\title{
Repression of ZCT1, ZCT2 and ZCT3 affects expression of terpenoid indole alkaloid biosynthetic and regulatory genes
}

\author{
Chun Yao Li ${ }^{1}$, Susan I Gibson ${ }^{\text {Corresp. } 1}$ \\ 1 Plant and Microbial Biology, University of Minnesota - Twin Cities, Saint Paul, MN, United States \\ Corresponding Author: Susan I Gibson \\ Email address: gibso043@umn.edu
}

Terpenoid indole alkaloids (TIAs) include several valuable pharmaceuticals. As Catharanthus roseus remains the primary source of these TIA pharmaceuticals, several research groups have devoted substantial efforts to increase production of these compounds by $C$. roseus. Efforts to increase TIA production by overexpressing positive regulators of TIA biosynthetic genes have met with limited success. This limited success might be due to the fact that overexpression of several positive TIA regulators turns on expression of negative regulators of TIA biosynthetic genes. Consequently, a more effective approach for increasing expression of TIA biosynthetic genes might be to decrease expression of negative regulators of TIA biosynthetic genes. Towards this end, an RNAi construct was generated that expresses a hairpin RNA carrying nucleotide fragments from three negative transcriptional regulators of TIA genes, ZCT1, ZCT2 and ZCT3, under the control of a beta-estradiol inducible promoter. Transgenic $C$. roseus hairy root lines carrying this ZCT RNAi construct exhibit significant reductions in transcript levels of all three ZCT genes. Surprisingly, out of eight TIA biosynthetic genes analyzed, seven (CPR, LAMT, TDC, STR, 16OMT, D4H and DAT) exhibited decreased rather than increased transcript levels in response to reductions in ZCT transcript levels. The lone exception was $T 19 H$, which exhibited the expected negative correlation in transcript levels with transcript levels of all three ZCT genes. A possible explanation for the $T 19 H$ expression pattern being the opposite of the expression patterns of the other TIA biosynthetic genes tested is that $\mathrm{T} 19 \mathrm{H}$ shunts metabolites away from vindoline production whereas the products of the other genes tested shunt metabolites towards vindoline metabolism. Consequently, both increased expression of $\mathrm{T} 19 \mathrm{H}$ and decreased expression of one or more of the other seven genes tested would be expected to have similar effects on flux through the TIA pathway. As $\mathrm{T19H}$ expression is lower in the ZCT RNAi hairy root lines than in the control hairy root line, the ZCTs could act directly to inhibit expression of $T 19 H$. In contrast, ZCT regulation of the other seven TIA biosynthetic genes tested is likely to occur indirectly, possibly by the ZCTs turning off expression of a negative transcriptional regulator of some TIA genes. 
In fact, transcript levels of a negative TIA transcriptional regulator, GBF1, exhibited a strong, and statistically significant, negative correlation with transcript levels of ZCT1, ZCT2 and ZCT3. Together, these findings suggest that the ZCTs repress expression of some TIA biosynthetic genes, but increase expression of other TIA biosynthetic genes, possibly by turning down expression of GBF1. 
1 Repression of ZCT1, ZCT2 and ZCT3 affects

2 expression of terpenoid indole alkaloid biosynthetic

3 and regulatory genes

4

5

6

7

8

9

10

11

12

13

14

15

16

17

18

19

20

21

22

23

24

25

26

27

28

29

30

31

32

33

34

35

36

37

38

39

Chun Yao Li ${ }^{1}$, Susan I Gibson ${ }^{1}$

${ }^{1}$ Plant and Microbial Biology, University of Minnesota - Twin Cities, Saint Paul, MN, USA

Corresponding Author:

Susan Gibson ${ }^{1}$

1500 Gortner Avenue, Saint Paul, MN 55108, USA

Email address: gibso043@umn.edu

\section{Abstract}

Terpenoid indole alkaloids (TIAs) include several valuable pharmaceuticals. As Catharanthus roseus remains the primary source of these TIA pharmaceuticals, several research groups have devoted substantial efforts to increase production of these compounds by C. roseus. Efforts to increase TIA production by overexpressing positive regulators of TIA biosynthetic genes have met with limited success. This limited success might be due to the fact that overexpression of several positive TIA regulators turns on expression of negative regulators of TIA biosynthetic genes. Consequently, a more effective approach for increasing expression of TIA biosynthetic genes might be to decrease expression of negative regulators of TIA biosynthetic genes. Towards this end, an RNAi construct was generated that expresses a hairpin RNA carrying nucleotide fragments from three negative transcriptional regulators of TIA genes, ZCT1, ZCT2 and ZCT3, under the control of a beta-estradiol inducible promoter. Transgenic $C$. roseus hairy root lines carrying this ZCT RNAi construct exhibit significant reductions in transcript levels of all three $Z C T$ genes. Surprisingly, out of eight TIA biosynthetic genes analyzed, seven (CPR, LAMT, $T D C, S T R, 16 O M T, D 4 H$ and $D A T)$ exhibited decreased rather than increased transcript levels in response to reductions in $Z C T$ transcript levels. The lone exception was $T 19 H$, which exhibited the expected negative correlation in transcript levels with transcript levels of all three $Z C T$ genes. A possible explanation for the $T 19 H$ expression pattern being the opposite of the expression patterns of the other TIA biosynthetic genes tested is that T19H shunts metabolites away from vindoline production whereas the products of the other genes tested shunt metabolites towards vindoline metabolism. Consequently, both increased expression of $\mathrm{T1} 9 \mathrm{H}$ and decreased expression of one or more of the other seven genes tested would be expected to have similar effects on flux through the TIA pathway. As $T 19 H$ expression is lower in the ZCT RNAi hairy root lines than in the control hairy root line, the ZCTs could act directly to inhibit expression of $T 19 H$. In contrast, ZCT regulation of the other seven TIA biosynthetic genes tested is likely to 
40

41

42

43

44

45

46

47

48

49

50

51

52

53

54

55

56

57

58

59

60

61

62

63

64

65

66

67

68

69

70

71

72

73

74

75

76

77

78

79

80

81

82

83

84

occur indirectly, possibly by the ZCTs turning off expression of a negative transcriptional regulator of some TIA genes. In fact, transcript levels of a negative TIA transcriptional regulator, $G B F 1$, exhibited a strong, and statistically significant, negative correlation with transcript levels of ZCT1, ZCT2 and ZCT3. Together, these findings suggest that the ZCTs repress expression of some TIA biosynthetic genes, but increase expression of other TIA biosynthetic genes, possibly by turning down expression of GBF1.

\section{Introduction}

Catharanthus roseus (L) G. Don produces several terpenoid indole alkaloids (TIAs), including vincristine, vinblastine, ajmalicine and serpentine, that are widely prescribed chemotherapeutics. As production of these chemicals in microbes and chemical synthesis are currently not feasible (van der Heijden et al., 2004; Shanks, 2005; Eastman, 2011), these chemicals continue to be harvested from the plant. Unfortunately, $C$. roseus produces these compounds in only very limited amounts. As a result, a number of research groups are characterizing the biosynthetic pathways leading to production of these chemicals and the regulatory pathways that help control flux through those pathways with the long-term goal of engineering plants to produce higher levels of these compounds.

The biochemical pathways leading to production of TIAs are complex (Fig. 1). The iridoid pathway catalyzes the synthesis of loganic acid from geranyl phosphate via multiple enzymatic steps (Miettinen et al., 2004). As part of this pathway, cytochrome P450 reductase (CPR) catalyzes the conversion of geraniol to 10-hydroxygeraniol (Meijer et al., 1993). The loganic acid is then converted to loganin via a reaction catalyzed by loganic acid-methyltransferase (LAMT) (Murata et al., 2008). A subsequent enzymatic reaction converts loganin to secologanin, one of the two precursors of TIA biosynthesis. The other precursor of TIA biosynthesis, tryptamine, is synthesized from tryptophan in a reaction catalyzed by tryptophan decarboxylase (TDC) (Goddijn et al., 1994). The enzyme strictosidine synthase (STR) then catalyzes the joining of secologanin and tryptamine to form strictosidine (Pasquali et al., 1992). Other TIAs are then synthesized from strictosidine via multiple, branching pathways. The enzymes 16-hydroxytabersonine-16-O-methyltransferase (16OMT) (Levac et al., 2008), desacetoxyvindoline 4-hydroxylase (D4H) (Vazquez-Flota et al., 1997) and deacetylvindoline acetyltransferase (DAT) (St-Pierre et al., 1998) catalyze reactions leading to the production of vindoline, which is then converted to vinblastine and vincristine via additional reactions. In contrast, tabersonine 19-hydroxylase (T19H) shunts metabolic flow away from production of vinblastine, by catalyzing the conversion of tabersonine to 19-hydroxytabersonine (Giddings et at., 2011).

Multiple transcriptional activators and repressors regulate the activity levels of TIA biosynthetic genes and genes from the TIA precursor pathways. ORCA2, an AP-2 domain protein, was the first transcriptional regulator of TIA biosynthetic genes identified from $C$. roseus. ORCA2 was originally shown to activate expression of STR (Menke et al., 1999). Overexpression of ORCA2 was subsequently shown to alter expression of several genes from the TIA biosynthetic pathway and both TIA precursor pathways (Li et al., 2013). A similar transcriptional regulator, ORCA3, was identified (van der Fits \& Memelink, 2000) and shown by several studies to alter expression of many of the genes in the TIA biosynthetic and TIA precursor pathways (Peebles et al., 2009;

Peer) reviewing PDF | (2021:01:57402:1:1:NEW 6 May 2021) 
85 Pan et al., 2012, Schweizer et al., 2018). The CrBPF1 transcriptional activator was identified via

86 a yeast one-hybrid screen using part of the STR promoter as bait (van der Fits et al., 2000).

87 However, overexpression of CrBPF1 has only modest effects on STR expression in C. roseus cell

88 suspension cultures (Zhang et al., 2011) and has no significant effects in C. roseus hairy root

89 cultures ( $\mathrm{Li}$ et al., 2015). In contrast, overexpression of CrBPF1 in hairy root cultures does

90 increase transcript levels for several genes from the TIA and both TIA feeder pathways (Li et al.,

91 2015). The BIS1 transcriptional activator turns on expression of all genes analyzed that encode

92 proteins in the metabolic pathway between geranyl diphosphate and loganic acid (Van

93 Moerkercke et al., 2015; Van Moerkercke et al., 2016; Schweizer et al., 2018). The CrMYC1

94 transcriptional activator was identified based on its ability to bind the STR promoter (Chatel et

95 al., 2003) and overexpression of CrMYC1 increases vinblastine, vincristine and catharanthine

96 levels (Sazegari et al., 2018). However, information regarding which TIA and TIA-related genes

97 may be regulated by CrMYC1 is currently lacking. In contrast, CrMYC2 has been shown to

98 regulate the expression of several TIA biosynthetic and regulatory genes (Zhang et al., 2011;

99 Schweizer et al., 2018). Similarly, overexpression of CrWRKY1 (Suttipanta et al., 2011) and

100 CrWRKY2 (Suttipanta, 2011) in C. roseus hairy root cultures has been shown to affect transcript

101 levels of several TIA biosynthetic and regulatory genes.

102

103

104

105

106

107

108

109

110

111

112

113

114

115

116

117

118

119

120

121

122

123

124

125

126

127

128

129

130

In addition to TIA transcriptional activators, several genes encoding TIA transcriptional repressors have been identified. GBF1 and GBF2 are transcriptional repressors that bind to elements in the STR and TDC gene promoters (Pré et al., 2000; Sibéril et al., 2001). ZCT1, ZCT2 and ZCT3 are TIA transcriptional repressors that were identified via a yeast one-hybrid screen using part of the TDC promoter as bait (Pauw et al., 2004). A comparison of the amino acid sequences of the ZCTs revealed that they share several motifs, including an L-box, B-box and LxLxL sequences (Pauw et al., 2004). All three ZCT proteins bind the TDC and STR promoters, although the exact binding sites within these promoters are different for ZCT1 and ZCT2 than for ZCT3. ZCT1, ZCT2 and ZCT3 suppress STR and TDC promoter activities in transactivation experiments and can also suppress the activities of ORCA2 and ORCA3 on the STR promoter (Pauw et al., 2004; Mortensen et al., 2019a). However, decreasing expression of ZCT1 and ZCT2 using an RNAi construct designed to reduce ZCT1 expression did not reduce expression of TDC, STR or G10H (Rizvi et al., 2016). ZCT1 and ZCT2, but not ZCT3, also repress expression of $H D S$, part of the methyl erythritol pathway (Chebbi et al., 2014). ZCT1 has also been shown to repress its own promoter (Mortensen et al., 2019b).

Several research groups have attempted to increase production of valuable TIAs by overexpressing TIA transcriptional activators. However, these efforts have generally met with limited success. For example, overexpression of ORCA2 led to initial increases in transcript levels of $P R X 1$, a major class III peroxidase that catalyzes the formation of $\alpha-3^{\prime}, 4^{\prime}-$ anhydrovinblastine (Costa et al, 2008). However, these increases were transient. In addition, overexpression of ORCA2 caused dramatic decreases, rather than increases, in DAT transcript levels (Li et al., 2013). A possible explanation for these findings is that some TIA transcriptional activators induce expression of TIA transcriptional repressors which then turn down expression of specific TIA biosynthetic genes. Consistent with this possibility are findings that overexpression of several TIA transcriptional activators turns on expression of TIA transcriptional repressors. For example, overexpression of either ORCA3 (Peebles et al., 2009) or ORCA2 (Li et al., 2013) causes increases in the transcript levels of all three ZCT genes, but

Peer) reviewing PDF | (2021:01:57402:1:1:NEW 6 May 2021) 
131 has no significant effects on expression of $G B F 1$ or $G B F 2$. Similarly, CrBPF1 overexpression 132 increases transcript levels of 11 out of 13 TIA transcriptional regulators assayed, including all 133 three $Z C T$ genes and both $G B F$ genes, although the effects on $G B F 2$ expression are relatively minor (Li et al., 2015). Overexpression of CrWRKY1 causes increased expression of all three ZCT genes, but not of GBF1 nor GBF2 (Suttipanta et al., 2011). In contrast, overexpression of CrWRKY2 in transgenic hairy roots causes substantial increases in expression of ZCT1 and ZCT3, but has only minor effects on expression of ZCT2, GBF1 and GBF2 (Suttipanta, 2011).

Findings that overexpression of TIA transcriptional activators often results in increased expression of TIA transcriptional repressors led to the suggestion that increased production of TIAs may require mechanisms designed to decrease expression of TIA repressors (Peebles et al., 2009). In an attempt to do this, researchers transformed C. roseus with an RNAi construct designed to turn off expression of ZCT1. This RNAi construct turns down expression of ZCT2 in addition to $Z C T 1$, but has no significant effects on $Z C T 3$ expression. $C$. roseus lines with this RNAi construct have no significant alterations in TIA levels, suggesting that reduced expression of all three ZCT genes may be necessary to increase TIA levels (Rizvi et al., 2016). Towards that end, in this study an RNAi construct designed to decrease expression of all three ZCT genes was designed and used to generate transgenic C. roseus hairy root lines.

\section{Materials \& Methods}

\section{Plant materials and growth conditions}

Catharanthus roseus, Vinca Little Bright Eye (www.neseed.com), was used for all of the experiments described. Seeds were placed in $50 \mathrm{~mL}$ falcon tubes and a solution consisting of $50 \%$ bleach and $0.02 \%$ triton X-100 was added to the seeds. The seeds were left in the solution for 7 to 8 minutes. The seeds were then washed with sterile water. Next, the seeds were germinated on Gamborg's B5 medium (Sigma, St. Louis, MO, USA) supplemented with Gamborg's vitamins (Sigma, St. Louis, MO, USA). Seeds were allowed to germinate in the dark for two weeks at $26^{\circ} \mathrm{C}$. The seedlings were then shifted to a 16 -h-light/8-h-dark cycle with a light intensity of approximately $44 \mu \mathrm{mol} \mathrm{m}{ }^{-2} \mathrm{~s}^{-1}$. After approximately 4 weeks of additional growth time, the seedlings were inoculated with Agrobacterium tumefaciens. The genes analyzed in this work are: 16OMT [GenBank: EF444544], BIS1 [GenBank: KM409646], CPR [GenBank: X69791], CrBPF1 [GenBank: AJ251686], CrMYC1 [GenBank: AF283506], CrMYC2 [GenBank: AF283507], CrWRKY1 [GenBank: HQ646368], CrWRKY2 [GenBank: JX241693], $D 4 H$ [GenBank: U71605], DAT [GenBank: AF053307], EF-1 [GenBank: EU007436], GBF 1 [GenBank: AF084971], GBF2 [GenBank: AF084972], LAMT [GenBank: EU057974], ORCA2 [GenBank: AJ238740], ORCA3 [GenBank: EU072424], STR [GenBank: X53602], T19H [GenBank: HQ901597], TDC [GenBank: X67662], UBQ11 [GenBank: EU007433], ZCT1 [GenBank: AJ632082], ZCT2 [GenBank: AJ632083] and ZCT3 [GenBank: AJ632084].

\section{Generation of a beta-estradiol inducible RNAi construct}

The pOpOff2(Hyg) RNAi vector, generously provided by CSIRO (Wielopolska et al., 2005), was modified by replacement of the dexamethasone-inducible promoter with a beta-estradiol inducible promoter from the pER8 XVE inducible system (Zuo, Niu \& Chua, 2000). The 
174 resulting vector was designed as the XVE-pOpOff2 RNAi vector. Fragments of the ZCT1, ZCT2

175

176

177

178

179

180

181

182

183

184

185

186

187

188

189

190

191

192

193

194

195

196

197

198

199

200

201

202

203

204

205

206

207

208

209

210

211

212

213

and ZCT3 genes that were 320-338 bp in length were amplified using KOD Hot Start DNA polymerase (Novagen, Madison, WI, USA) and genomic DNA from C. roseus variety Little Bright Eye. The oligonucleotides used for these PCR reactions were as follows: ZCT1, 5' TGGAGAAATCAGCAGTACCGGCGT 3' paired with 5' TGGTACCGCCTTTGCAACAGG 3'; ZCT2, 5' TACCGATGAAGCGTACGAGA 3' paired with 5' ACCTCCGAGAGCTTGACCGATAGC 3' and ZCT3, 5' ACGAAAACGCAGCTACTCTCCGCT 3' paired with 5' TGCCTTATGTCCTCCGAGTGCTTGG 3'. Oligonucleotides 5' GTTGCAAAGGCGGTACCATACCGATGAAGCGTACGAG 3' and 5' GGTCAAGCTCTCGGAGGTACGAAAACGCAGCTACTC 3' were used to run a bridge PCR to combine the ZCT1, ZCT2 and ZCT3 DNA fragments into one larger nucleotide fragment. The ZCT1/2/3 fragment was cloned into the PCR8/GW/topo entry vector (Invitrogen, Grand Island, NY, USA) and then transferred to the XVE-pOpOff2 RNAi vector through the LR reaction using LR clonase mix II (Invitrogen, Grand Island, NY, USA). The resulting construct, designated XVE-pOpOff2-ZCT (XPZ), contains two copies of the ZCT1/2/3 DNA fragment. The two $Z C T 1 / 2 / 3$ sequences are in an inverted repeat orientation and are separated by the DNA spacer present in the original pOpOff2(Hyg) RNAi vector (Fig. S1). The XPZ construct was then used for transformation of Agrobacterium tumefaciens strain GV3101.

\section{Generation of transgenic hairy roots}

Approximately 6-weeks old C. roseus seedlings were used for the plant transformation procedure. Transformation experiments were carried out as previously described (Li et al., 2013). In particular, transformation was achieved using an approximately equal mixture of $A$. tumefaciens strain GV3101 cultures transformed with the XPZ RNAi construct or with the pPZPROL plasmid. The pPZPROL plasmid carries the rol $A B C$ genes, which have been shown to be sufficient to induce formation of hairy roots on C. roseus. A. tumefaciens strain GV3101 carrying the rol $A B C$ genes was used for these experiments because the hairy roots produced in this way tend to show better adaptability to growth in liquid culture than those produced using $A$. rhizogenes (Hong et al., 2006). Hairy roots appeared on inoculation sites approximately 4 weeks later. After the hairy roots reached lengths of approximately $1 \mathrm{~cm}$, they were excised and transferred to solid medium supplemented with $30 \mathrm{~g} \mathrm{~L}^{-1}$ sucrose, $6 \mathrm{~g} \mathrm{~L}^{-1}$ agar, $250 \mathrm{mg} \mathrm{L}^{-1}$ cefotaxime, half-strength Gamborg's B5 salts and full-strength Gamborg's vitamins ( $\mathrm{pH}$ 5.8). After one week of growth on solid media, $30 \mathrm{mg} \mathrm{L}^{-1}$ hygromycin was used to select for hairy roots carrying the XPZ construct. Hairy roots carrying the XPZ construct were transferred to 50 $\mathrm{mL}$ of liquid media, comprised of $50 \mathrm{~mL}$ of half-strength Gamborg's B5 liquid solution supplemented with full-strength Gamborg's vitamins and $30 \mathrm{~g} \mathrm{~L}^{-1}$ sucrose. The flasks containing the transgenic hairy roots were kept on a shaker at $225 \mathrm{rpm}$ in the dark and were sub-cultured every 5 weeks. To confirm that these hairy roots carried the XPZ RNAi construct, pcr was used to amplify sequences from the ZCT1 to ZCT3 fragments carried on the XPZ RNAi construct,

Peer] reviewing PDF | (2021:01:57402:1:1:NEW 6 May 2021) 
214 from the T-DNA right border to the ZCTI fragment and from the ZCT3 fragment to the XVE

215 sequences. The results of these pcr experiments confirmed the presence of the XPZ RNAi

216 construct (data not shown). To generate a negative control line, C. roseus was also transformed

217 with XVE-pOpOff2 empty vector, using the same procedure described above for transformation

218 with the XPZ RNAi construct.

219

220

221

\section{Induction of transgene expression and tissue collection}

222

Induction of expression of the RNAi construct was carried out was carried out largely as described previously (Li et al., 2013). Specifically, to induce expression of the ZCT1/2/3 hairpin

223 sequence, three actively growing hairy roots, each 3 to $4 \mathrm{~cm}$ in length, were transferred to a 250

224 $\mathrm{mL}$ flask containing $50 \mathrm{~mL}$ of half-strength B5 media. The cultures were grown on a shaker at

225

226

227 $100 \mathrm{rpm}$ in the dark for 31 days. The media was replaced with fresh half-strength B5 on days 17 and 28. On day 31 expression of the RNAi construct was induced by addition of $50 \mu \mathrm{L}$ of 20

228 $\mathrm{mM}$ beta-estradiol (in ethanol) to the liquid culture, for a final concentration of $20 \mu \mathrm{M}$ betaestradiol. As a control, un-induced cultures were treated with $50 \mu \mathrm{L}$ of ethanol at the same time. The hairy root cultures were returned to a dark environment. Cultures were harvested $0,6,12$, 24, 48 and $72 \mathrm{~h}$ after the start of induction. Hairy roots transformed with the XVE-pOpOff2 empty vector were used as a negative control and were treated the same way as the hairy roots

233

234

235

236

237

238

239

240

241

242

243

244

245

246

247

248

249

250

251

252 transformed with the XPZ RNAi construct. Three independent hairy root cultures were harvested for each transgenic hairy root line, time point and media combination. Upon collection, hairy root samples were immediately flash frozen in liquid nitrogen and then stored at $-80^{\circ} \mathrm{C}$ prior to being used for gene expression analyses.

\section{RNA extraction and RT-qPCR analyses}

Total RNA was extracted as previously described (Li et al., 2013) using the Spectrum Total RNA Isolation Kit (Sigma, St. Louis, MO, USA) with on-column DNase I digestion. cDNAs were synthesized using goscript reverse transcriptase (Promega, Madison, WI, USA). Gene transcript levels were analyzed by qPCR using the SYBR Premix EX Taq II (2X) (Tli RNase H plus (Clontech Laboratories, Mountain View, CA, USA) and reaction were run on a Roche LightCycler 480 II. For all experiments qPCR data were normalized using the geometric average of qPCR results for two control genes, EF1 and UBQ11, which were shown previously to be the two most stably expressed genes of those tested in C. roseus (Wei, 2010). For statements of fold changes in transcript levels, a change of one Ct (i.e., a change of one PCR cycle) was estimated to represent a two-fold change in transcript levels. Relative mRNA levels are expressed as $\Delta \Delta \mathrm{Ct}$. $\Delta \Delta \mathrm{Ct}=\Delta \mathrm{Ct}_{\text {un-induced control line at } 0 \mathrm{~h}}-\Delta \mathrm{Ct}_{\text {other }} . \Delta \mathrm{Ct}_{\text {un-induced control line at } 0 \mathrm{~h}}=\mathrm{Ct}_{\text {indicated gene in un-induced control }}$ line at $0 \mathrm{~h}-\mathrm{CT}_{\mathrm{EF} 1 / \mathrm{UBQ} 11 \text { in un-induced control line at } 0 \mathrm{~h} .} \Delta \mathrm{Ct}_{\text {other }}=\mathrm{Ct}_{\text {indicated gene }}-\mathrm{Ct}_{\mathrm{EF} 1 / \mathrm{UBQ} 11}$ for the time point, line and growth condition being analyzed.

\section{Statistical analyses}


253 A two-tailed Student's T-test was employed to determine statistical significance between

254 induced and un-induced cultures of the same hairy root line at the same timepoint, or between an

255 RNAi line and the control line grown on the same media for the same amount of time. The

256 Pearson product moment correlation coefficient was used to identify correlations between

257 expression levels of different pairs of genes across all hairy root lines, culture conditions and

258 timepoints analyzed. The significance of the Pearson product moment correlation coefficient was

259 determined using the $p$ value calculator at:

260 https://www.socscistatistics.com/pvalues/pearsondistribution.aspx.

261

\section{Results and Discussion}

$263 Z \boldsymbol{Z C T}$ expression is significantly decreased in RNAi hairy root lines

264 The ZCT1, ZCT2 and ZCT3 transcriptional regulators have been shown to act as negative

265 regulators of genes involved in TIA metabolism and regulation of TIA metabolism (Pauw et al.,

266 2004; Chebbi et al., 2014; Mortensen et al., 2019a; Mortensen et al., 2019b). To investigate the

267 role of the ZCTs in these processes, it was desirable to obtain C. roseus hairy root lines with

268 decreased expression of all of the ZCTs. Towards this end, an RNAi construct was generated that

269 is designed to reduce expression of all three of the ZCT genes. This RNAi construct, designated

$270 \mathrm{XPZ}$, carries inverted repeats of a DNA sequence that contains 320-338 bp fragments from each

271 of the three $Z C T$ genes. These sequences are designed to be expressed under the control of a

272 beta-estradiol inducible promoter (Zuo, Niu \& Chua, 2000) in the XVE-pOpOff2 vector. An

273 inducible promoter was used for these experiments as there was a concern that constitutive

274 repression of the $Z C T$ genes could be harmful to the growth of transgenic hairy roots. The XPZ

275 construct was used to generate C. roseus transgenic hairy root lines. Based on preliminary testing 276 of transgene expression levels, two lines (XPZ28 and XPZ38) were chosen for further analysis.

277 A negative control line (C) was generated using the XVE-pOpOff2 empty vector.

278

279 To test the effects of the XPZ construct on ZCT gene expression, two hairy root lines carrying 280 the XPZ construct (XPZ28 and XPZ38) and one control line (C) were grown in the presence or 281 absence of the inducer of the XPZ RNAi construct, beta-estradiol. Cultures were harvested 0,6 , $28212,24,48$ and $72 \mathrm{~h}$ after addition of 0 or $20 \mu \mathrm{M}$ beta-estradiol to the cultures. Analysis of ZCTI, $283 Z C T 2$ and ZCT3 mRNA levels in tissues harvested from these cultures revealed that expression 284 of all three genes was significantly reduced in the XPZ28 and XPZ38 lines compared to the 285 control line (Fig. 2). At the zero timepoint, ZCT1 expression was down 13 fold in the XPZ28 line 286 relative to the control line and 29 fold in the XPZ38 line (Fig. 2A). Similarly, expression of 287 ZCT2 was down 39 fold and 34 fold in the XPZ28 and XPZ38 lines, respectively (Fig. 2B). 288 Expression of the $Z C T 3$ gene was decreased to a lesser, but still significant extent, being down 289 six and four fold in the XPZ28 and XPZ38 lines, respectively (Fig. 2C). These results indicate 290 that the RNAi construct causes a significant reduction in expression of all three ZCT genes, even 291 in the absence of the inducing agent for the construct. The most likely explanation for this 292 finding is that expression of the RNAi construct may be leaky, with substantial expression 
293

294

295

296

297

298

299

300

301

302

303

304

305

306

307

308

309

310

311

312

313

314

315

316

317

318

319

320

321

322

323

324

325

326

327

328

329

330

331

332

occurring even in the absence of the inducing agent. The transgenic RNAi lines grew well, alleviating concerns that constitutive repression of ZCT expression could have deleterious effects on growth of hairy root cultures.

Although expression of the ZCT genes was significantly decreased in both RNAi hairy root lines prior to the addition of beta-estradiol, growth on beta-estradiol did result in further reductions in expression of all three ZCT genes. For example, at the 12-hour timepoint ZCT1 expression was down approximately seven fold (Fig. 2A), ZCT2 expression was down approximately four fold (Fig. 2B) and ZCT3 expression was down three to four fold (Fig. 2C) in the RNAi lines grown on $20 \mu \mathrm{M}$ beta-estradiol relative to the same lines grown on $0 \mu \mathrm{M}$ beta-estradiol. When the RNAi lines growing on $20 \mu \mathrm{M}$ beta-estradiol were compared with the control line growing on $20 \mu \mathrm{M}$ beta-estradiol, the reductions in ZCT expression in the RNAi lines were quite large and were statistically significant at all timepoints tested. For example, after growth on $20 \mu \mathrm{M}$ betaestradiol for 12 hours, expression of all three ZCT genes was down by approximately 20 to 80 fold in both RNAi lines relative to the control line.

\section{Decreased expression of the $Z C T$ genes results in altered expression of TIA biosynthetic genes}

As the $Z C T$ genes have been shown to act as negative regulators of transcription, decreased expression of the ZCT genes was expected to result in increased expression of at least some TIA biosynthetic and regulatory genes. To test this hypothesis, transcript levels of genes from both the monoterpenoid and indole feeder pathways and from the TIA pathways were analyzed. A time course was performed for these experiments as several previous studies have shown that the effects of altering expression of regulatory genes on expression of TIA biosynthetic and regulatory genes are often transitory (Costa et al., 2008; Peebles et al., 2009; Li et al., 2013, 2015). Therefore, it was desirable to assay several timepoints to identify as many regulated genes as possible. However, the leakiness of the beta-estradiol promoter resulted in substantial levels of $Z C T$ repression even in the absence of the inducing agent. In fact, as seen below, many of the genes found to be regulated by the ZCTs exhibit approximately equal levels of regulation at the 0 $\mathrm{h}$ timepoint as at later timepoints (i.e., after addition of the inducing agent). These results suggest that many of the observed effects might be due to long-term repression of ZCT expression. It is possible that short-term repression of ZCT expression could have somewhat different effects on expression of TIA regulatory and biosynthetic genes.

Surprisingly, expression of the CPR (Fig. 3A) and LAMT (Fig. 3B) genes from the monoterpenoid pathway was found to be reduced, rather than increased, in the RNAi lines relative to the control line. Although the decreases in $C P R$ transcript levels were quite modest, reaching a maximum of a five-fold reduction in the XPZ38 line relative to the control line after growth on $20 \mu \mathrm{M}$ beta-estradiol for $48 \mathrm{~h}$, they were statistically significant at most timepoints analyzed for the XZP38 line (Fig. 3A). In addition, when comparing CPR expression levels with 
333

334

335

336

337

338

339

340

341

342

343

344

345

346

347

348

349

350

351

352

353

354

355

356

357

358

359

360

361

362

363

364

365

366

367

368

369

370

371

372

ZCT1, ZCT2 or ZCT3 expression levels in all hairy root lines, timepoints and media analyzed, a statistically significant positive correlation was found between $C P R$ expression and expression of each of the ZCT genes (Table 1). Similarly, LAMT transcript levels were reduced by approximately three to four fold in the RNAi lines compared to the control line at the same timepoint and beta-estradiol concentration (Figure 3B). Determination of the Pearson product moment correlation coefficient for $L A M T$ expression and expression of each of the ZCT genes also revealed a statistically significant positive correlation between $L A M T$ expression and expression of each of the ZCT genes (Table 1).

TDC catalyzes the last step in the indole pathway leading to production of one of the two TIA precursors, tryptamine (Goddijn et al., 1994). On average, decreased expression of the ZCT genes in the RNAi lines caused a small reduction in TDC transcript levels (Fig. 4). Reductions in $T D C$ transcript levels were particularly modest in the XPZ28 line, being statistically significant at only the 6- and 12-h timepoints in the cultures growing on $0 \mu \mathrm{M}$ beta-estradiol and at the 12-h timepoint for the cultures growing on $20 \mu \mathrm{M}$ beta-estradiol. The XPZ38 line exhibited a greater reduction in $T D C$ transcript levels. TDC expression was significantly decreased in the XPZ38 line on both media and at all timepoints tested, with the exception of the 24-h timepoint for cultures growing on $20 \mu \mathrm{M}$ beta-estradiol (Fig. 4). Although TDC expression was decreased less than two fold on average in the XPZ28 line and between two and three fold in the XPZ38 line, analysis of the Pearson correlation coefficient for TDC expression levels and ZCT1, ZCT2 or $Z C T 3$ expression levels revealed that there is a statistically significant positive correlation between $T D C$ expression and expression of all three ZCT genes (Table 1).

To determine whether reductions in ZCT expression levels can affect expression of TIA biosynthetic genes, transcript levels for several TIA biosynthetic genes were analyzed in the ZCT RNAi and control lines (Fig. 5). STR catalyzes the first step in TIA biosynthesis, namely the combination of secologanin and tryptamine to form strictosidine (Pasquali et al., 1992).

Decreased expression of the ZCT genes had a minor, negative effect on STR transcript levels. Reductions in STR transcript levels were statistically significant at only a few of the combinations of hairy root lines, media and timepoints tested (Fig. 5A). However, analysis of the Pearson correlation coefficient for STR expression levels and ZCT1, ZCT2 or ZCT3 expression levels revealed that there is a statistically significant positive correlation between STR expression and expression of all three of the ZCT genes (Table 1). T19H catalyzes the conversion of tabersonine to 19-hydroxytabersonine (Giddings et at., 2011). Interestingly, decreased expression of the ZCT genes caused an average increase in $T 19 H$ expression (Fig. 5B). Increases in $\mathrm{T} 19 \mathrm{H}$ transcript levels averaged only approximately $50 \%$ for both RNAi lines, and were statistically significant for only a few of the combinations of hairy root line, media and timepoint tested. However, there was a statistically significant negative correlation between $\mathrm{T} 19 \mathrm{H}$ transcript levels and expression levels of all three ZCT genes when analyzing all of the hairy root lines, media and timepoints analyzed (Table 1). In contrast, reductions in ZCT expression levels 
373 caused a decrease in 16OMT expression levels (Fig. 5C). 16OMT catalyzes the conversion of 16374 hydroxytabersonine to 16-methoxytabersonine (Levac et al., 2008). 16OMT expression levels 375 were down an average of 1.5 fold in the XPZ28 line and 2.5 fold in the XPZ38 line. Analysis of 376 the Pearson correlation coefficient for $16 O M T$ expression and expression of the ZCT1, ZCT2 or $377 Z C T 3$ genes revealed a statistically significant positive correlation between $16 O M T$ and $Z C T$ 378 expression levels. Similarly, expression of the $D 4 H$ gene was down an average of approximately 379 two or three fold in the XPZ28 and XPZ38 lines, respectively, relative to the control line (Fig. 380 5D). D4H catalyzes the conversion of desacetoxyvindoline to deacetylvindoline (Vazquez-Flota

381

382

383

384

385

386

387

388

389

390

391

392

393

394

395

396

397

398

399

400

401

402

403

404

405

406

407

408

409

410

411

412 et al., 1997). Decreased expression of the ZCT genes also resulted in an average decrease of approximately four fold in $D A T$ expression in both RNAi lines (Fig. 5E). DAT catalyzes the conversion of deacetylvindoline to vindoline (St-Pierre et al., 1998). Analyses of Pearson correlation coefficients between expression of $D 4 H$ or DAT and ZCT1, ZCT2 or ZCT3 revealed a statistically significant positive correlation between expression of $D 4 H$ and $D A T$ and expression of each of the ZCT genes (Table 1).

As the $Z C T$ genes have previously been characterized as negative transcriptional regulators, decreased expression of the ZCT genes was anticipated to lead to increased expression of at least some TIA biosynthetic genes. Surprisingly, seven of the eight biosynthetic genes tested exhibited decreased rather than increased expression in response to reductions in ZCT transcript levels. The lone exception was $T 19 H$, which exhibited the expected negative correlation in transcript levels with $Z C T$ transcript levels. A possible explanation for the $T 19 H$ expression pattern being the opposite of the expression patterns of the other biosynthetic genes tested is that $\mathrm{T} 19 \mathrm{H}$ shunts metabolites away from vindoline production whereas the other genes tested shunt metabolites towards vindoline metabolism (Fig. 1). Consequently, both increased expression of $\mathrm{T1} 9 \mathrm{H}$ and decreased expression of one or more of the other seven genes tested would be expected to have similar effects on flux through different branches of the TIA pathway. For example, both increased expression of $T 19 H$ and decreased expression of one of the other seven TIA biosynthetic genes would be expected to result in decreased flux from tabersonine to vindoline.

\section{Decreased expression of the $Z C T$ genes results in altered expression of TIA regulatory genes}

Surprisingly, decreased expression of the ZCT genes led to decreased expression of seven of the eight biosynthetic genes analyzed (Fig. 5). As the ZCT genes have previously been characterized as negative regulators of transcription, a possible explanation for this result is that the ZCTs affect expression of some of the TIA biosynthetic genes indirectly. For example, it is possible that the ZCTs cause decreased expression of one or more of the other negative regulators of expression of TIA biosynthetic genes. In that case, decreased expression of the ZCT genes could lead to increased expression of those negative regulator(s) and, consequently, decreased expression of the biosynthetic genes regulated by those other negative regulators. As decreased expression of the ZCT genes led to increased expression of $T 19 H$, the ZCTs could be affecting 
$413 T 19 H$ expression directly or indirectly. Direct repression of $T 19 H$ expression could be caused, 414 for example, by one or more of the ZCTs binding to a regulatory sequence in the $T 19 H$ promoter 415 and interfering with transcription of $T 19 H$. Alternatively the ZCTs could affect $T 19 H$ expression 416 levels indirectly by repressing expression of a positive regulator of $T 19 H$ expression.

417

418 Additional analyses were done to test these possible mechanisms for ZCT regulation of TIA 419 biosynthetic genes. The expression levels of eight genes (BIS1, CrBPF1, CrMYC1, CrMYC2,

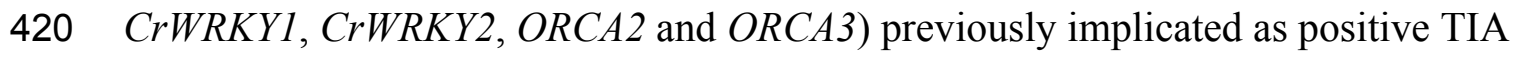
421 transcriptional regulators and of two genes $(G B F 1$ and $G B F 2)$ previously implicated as negative 422 TIA transcriptional regulators were determined. Expression of ZCT3, but not of ZCT1 or ZCT2, 423 exhibited a statistically significant negative correlation with expression of $O R C A 2$. In contrast, $424 Z C T 2$, but not ZCT1 or ZCT3, exhibited a statistically significant negative correlation with expression of ORCA3 (Table 1). However, the effects of ZCT3 and ZCT2 on expression of ORCA2 (Fig. 6A) and ORCA3 (Fig. 6B) were of limited magnitude. BIS1 transcript levels are

427

428

429

430

431

432

433

434

435

436

437

438

439

440

441

442

443

444

445

446

447

448

449

450

451

452 lower in the RNAi lines than in the control line at most timepoints tested (Fig. 6C). Although this is especially true for the XPZ38 line, even the XPZ38 exhibited only modest differences in BIS1 transcript levels relative to the control line. However, expression of all three ZCT genes exhibited statistically significant positive correlations with BIS1 transcript levels (Table 1). In the case of CrBPF 1, ZCT expression levels generally had little effect on CrBPF1 transcript levels (Fig. 6D), although $Z C T 2$ transcript levels did exhibit a statistically significant positive correlation with CrBPF1 transcript levels (Table 1). Decreased ZCT expression tended to lead to decreased CrMYC1 expression, especially for the XPZ38 line grown on media containing $20 \mu \mathrm{M}$ betaestradiol (Fig. 6E). In addition, expression levels of all three ZCT genes showed statistically significant positive correlations with CrMYC1 transcript levels (Table 1). In contrast, ZCT expression levels had very little effect on $C r M Y C 2$ transcript levels (Fig. 6F) and there were no statistically significant correlations between $C r M Y C 2$ transcript levels and the transcript levels of any of the ZCT genes (Table 1). ZCT expression levels exhibited minor and inconsistent effects on $C r W R K Y 1$ transcript levels, although there was a tendency for $C r W R K Y 1$ transcript levels to be slightly decreased in the RNAi lines (Fig. 6G) and there was a statistically significant positive correlation between $C r W R K Y 1$ transcript levels and transcript levels of each of the ZCT genes (Table 1). CrWRKY2 transcript levels tended to be down in the XPZ38 line, but exhibited inconsistent results for the XPZ28 line (Fig. 6H). Transcript levels of ZCT1, but not of ZCT2 or ZCT3, exhibited a statistically significant positive correlation with $C r W R K Y 2$ transcript levels (Table 1).

The strongest effects on expression of a TIA transcriptional regulator were observed for the negative transcriptional regulator, $G B F 1$. In line XPZ28, GBF1 transcript levels were typically two- to 10-fold higher than in the control line at the same timepoint and grown on the same media. For line XPZ38, GBF1 transcript levels were typically 10- to 30-fold higher than in the control line at the same timepoint and grown on the same media (Fig. 7A). In addition, the 
453 transcript levels of all three $Z C T$ genes exhibited strong, statistically significant negative 454 correlations with $G B F 1$ transcript levels (Table 1). Interestingly, $Z C T$ transcript levels had the 455 opposite effect on expression of $G B F 2$ as on expression of $G B F 1$, as $G B F 2$ transcript levels 456 tended to be slightly lower in the RNAi lines than in the control line grown on the same media 457 (Fig. 7B). Although the effects of $Z C T$ transcript levels on GBF2 transcript levels were relatively 458 minor, the expression levels of all three $Z C T$ genes did exhibit statistically significant positive

459

460

461

462

463

464

465

466

467

468

469

470

471

472

473

474

475

476

477

478

479

480

481

482

483

484

485

486

487

488

489

490

491

492 correlations with GBF2 transcript levels (Table 1). However, because GBF2 transcript levels were down an average of only approximately $12 \%$ in the induced XPZ28 cultures and $26 \%$ in the induced XPZ38, cultures, GBF1 is likely to play a much more important role in regulation of TIA biosynthetic gene expression than does GBF2.

The positive correlations observed between ZCT transcript levels and transcript levels of seven of the eight TIA biosynthetic genes analyzed could be explained by the ZCTs negatively regulating expression of one or more of the other negative regulators of TIA biosynthetic genes. Besides the ZCTs, GBF1 and GBF2 have been postulated to act as negative regulators of some TIA biosynthetic genes (Pré et al., 2000; Sibéril et al., 2001). Interestingly, GBF1 transcript levels were found to be much higher in both of the ZCT RNAi lines than in the control line and the Pearson product moment correlation coefficient showed a statistically significant negative correlation for transcript levels for all three of the ZCT genes and GBF1 transcript levels (Table 1). These findings suggest a model where all three of the ZCTs act as negative regulators of $G B F 1$ expression and GBF1, in turn, acts as a negative regulator of expression of $C P R, L A M T$, $T D C, S T R, 16 O M T, D 4 H$ and $D A T$. The available data do not indicate whether the effects of the ZCTs on GBF1 expression, and the effects of GBF1 on expression of the TIA biosynthetic genes, are direct or indirect. For example, the ZCTs could bind to elements in the $G B F 1$ promoter and directly repress expression of $G B F 1$. Alternatively, the ZCTs could reduce $G B F 1$ expression by repressing expression of a positive regulator that is required for $G B F 1$ expression. Among the positive regulators of TIA genes tested, only expression of ORCA2 and ORCA3 were found to exhibit a negative correlation with expression of a $Z C T$ gene. Expression of $O R C A 2$ was negatively correlated with $Z C T 3$ expression and expression of $O R C A 3$ was negatively correlated with ZCT2 expression (Table 1). These findings raise the possibility that ZCT2 and ZCT3 could affect $G B F 1$ expression indirectly by repressing expression of $O R C A 3$ and $O R C A 2$, respectively. However, previous results indicating that neither ORCA2 (Li et al., 2013) nor ORCA3 (Peebles et al., 2009) regulate $G B F 1$ transcript levels argue against the possibility that the ZCTs regulate $G B F 1$ indirectly via effects on $O R C A 2$ and/or $O R C A 3$ expression.

GBF1 could act directly to repress expression of specific TIA biosynthetic genes by binding elements in their promoters, thereby inhibiting expression of those genes. Alternatively, GBF1 could inhibit expression of a positive regulator that is required for expression of the TIA biosynthetic genes. Among the positive regulators of TIA gene expression tested, BIS1, CrMYCl and $C r W R K Y 1$ exhibit statistically significant positive correlations with expression levels of all 
493 three $Z C T$ gene (Table 1). These findings raise the possibility that GBF1 could repress

494 expression of some TIA biosynthetic genes by turning off expression of $B I S 1, C r M Y C 1$ and/or

$495 \mathrm{CrWRKY1}$. However, previous research findings indicate that BIS1 does not affect expression of

496 TDC, STR or T19H (Schweizer et al., 2018), indicating that GBF1 does not regulate expression

497 of at least those TIA genes via effects on BISI expression levels. In contrast, CrMYC1 has been

498 shown to bind the STR promoter (Chatel et al., 2003), leaving open the possibility that GBF1

499 could help regulate expression of some TIA biosynthetic genes via effects on CrMYC1 activity

500 levels. CrWRKY1 acts as a positive regulator of TDC, but not of CPR or STR (Suttipanta et al.,

501 2011). Consequently, it is possible that GBF1 could regulate expression of some, but not all, TIA

502 biosynthetic genes via effects on CrWRKY1 expression levels.

503

504 Conclusions

505 Two C. roseus transgenic lines expressing the XPZ RNAi construct exhibit significant decreases

506 in transcript levels of ZCT1, ZCT2 and ZCT3. As the ZCTs are believed to function as repressors

507 of transcription, decreased expression of the ZCTs may be expected to result in increased

508 expression of genes regulated by the ZCTs. However, of eight genes from the TIA and TIA-

509 feeder pathways tested, only expression of $T 19 H$ increased in the ZCT RNAi lines relative to the

510 control line. This finding raises the possibility that the ZCTs act directly to inhibit expression of

$511 \mathrm{T19H}$, although it is also possible that the ZCTs could act by turning down expression of a

512 positive regulator of $T 19 H$ expression. In contrast, expression of the other seven TIA

513 biosynthetic genes tested exhibited a positive correlation with the expression levels of all three

$514 Z C T$ genes (Table 1). These findings suggest that the ZCTs affect regulation of these genes

515 indirectly, possibly by turning off expression of a negative transcriptional regulator of these

516 genes. In fact, $G B F 1$ transcript levels are much lower in the control transgenic hairy root lines

517 than in the RNAi lines. This finding suggests a model where all three of the ZCTs act as negative

518 regulators of $G B F 1$ expression and GBF1, in turn, acts as a negative regulator of expression of

519 CPR, LAMT, TDC, STR, 16OMT, D4H and DAT.

520

521

\section{Acknowledgements}

522

Mr. Alex Leopold is thanked for assistance in generating and maintaining the C. roseus

523 transgenic hairy root lines used in these studies. Prof. Jackie Shanks (Iowa State University) is

524 thanked for many interesting and insightful discussions.

525

526

References 
528 G. Chatel, G. Montiel, M. Pré, J. Memelink, M. Thiersault, B. Saint-Pierre, P. Doireau, P.

529 Gantet, CrMYC1, a Catharanthus roseus elicitor- and jasmonate-responsive bHLH transcription

530 factor that binds the G-box element of the strictosidine synthase gene promoter, Journal of

531 Experimental Botany 54 (2003) 2587-2588.

532

533 M. Chebbi, O. Ginis, V. Courdavault, G Glévarec, A. Lanoue, M. Clastre, N. Papon, C. Gaillard, 534 R. Atanassova, B. St-Pierre, N. Giglioli-Guivarc'h, M. Courtois, A. Oudin, ZCT1 and ZCT2 535 transcription factor repress the activity of a gene promoter from the methyl erythritol phosphate 536 pathway in Madagascar periwinkle cells, Journal of Plant Physiology 171 (2014) 1510-1513. 537

538 M.M.R. Costa, F. Hilliou, P. Duarte, L.G. Pereira, I. Almeida, M. Leech, J. Memelink, A. Ros 539 Barceló, M. Sottomayor, Molecular cloning and characterization of a vacuolar class III 540 peroxidase involved in the metabolism of anticancer alkaloids in Catharanthus roseus, Plant 541 Physiology 146 (2008) 403-417. 
542

543 P. Eastman, Oncology drug shortages worsening, threatening patient care, Oncology Times 33

544 (2011) 14-15.

545

546 L.-A. Giddings, D.K. Liscombe, J.P. Hamilton, K.L. Childs, D. DellaPenna, C.R. Buell, S.E.

547 O'Connor, A stereoselective hydroxylation step of alkaloid biosynthesis by a unique cytochrome

548 P450 in Catharanthus roseus, Journal of Biological Chemistry 286 (2011) 16751-16757.

549

550 O.J.M. Goddijn, F.P. Lohman, R.J. de Kam, R.A. Hilperoort, J.H.C. Hoge, Nucleotide sequence

551 of the tryptophan decarboxylase gene of Catharanthus roseus and expression of tdc-gusA gene

552 fusions in Nicotiana tabacum, Molecular and General Genetics 242 (1994) 217-225.

553

554 S.B. Hong, C.A.M. Peebles, J.V. Shanks, K.Y. San, S.I. Gibson, Terpenoid indole alkaloid

555 production by Catharanthus roseus hairy roots induced by Agrobacterium tumefaciens harboring 556 the rol ABC genes, Biotechnology and Bioengineering 93 (2006) 386-390.

557

558

559

D. Levac, J. Murata, W.S. Kim, V. De Luca, Application of carborundum abrasion for

560 investigating the leaf epidermis: molecular cloning of Catharanthus roseus 16-

561

562 hydroxytabersonine-16-O-methyltransferase, The Plant Journal 53 (2008) 225-236.

563

C.Y. Li, A.L. Leopold, G.W. Sander, J.V. Shanks, L. Zhao, S.I. Gibson, The ORCA2

564 transcription factor plays a key role in regulation of the terpenoid indole alkaloid pathway, $B M C$

565

566 Plant Biology 13 (2013) 155.

567 C.Y. Li, A. Leopold, G.W. Sander, J.V. Shanks, L. Zhao, S.I. Gibson, CrBPF1 overexpression alters transcript levels of terpenoid indole alkaloid biosynthetic and regulatory genes, Frontiers

568 in Plant Science 6 (2015) 818.

569

570 D.-H. Liu, W.-W. Ren, L.-J. Cui, L.-D. Zhang, X.-F. Sun, K.-X. Tang, Enhanced accumulation

571 of catharanthine and vindoline in Catharanthus roseus hairy roots by overexpression of

572 transcriptional factor ORCA2, African Journal of Biotechnology 10 (2011) 3260-3268.

573

574 A.H. Meijer, M.I. Lopes Cardoso, J.T. Voskuilen, A. De Waal, R. Verpoorte, J.H.C. Hoge, Isolation and characterization of a cDNA clone from Catharanthus roseus encoding

576 NADPH:cytochrome $\mathrm{P} 450$ reductase, an enzyme essential for reactions catalysed by cytochrome P450 mono-oxygenases in plants, The Plant Journal 4 (1993) 47-60.

578 
579 F.L. Menke, A. Champion, J.W. Kijne, J. Memelink, A novel jasmonate- and elicitor-responsive 580 element in the periwinkle secondary metabolite biosynthetic gene Str interacts with a jasmonate581 and elicitor-inducible AP2-domain transcription factor, ORCA2, The EMBO Journal 18 (1999) $582 \quad 4455-4463$.

583

584 K. Miettinen, L. Dong, N. Navrot, T. Schneider, V. Burlat, J. Pollier, L. Woittiez, S. van der 585 Krol, R. Lugan, T. Ilc, R. Verpoorte, K.-M. Oksman-Caldentey, E. Martinoia, H. Bouwmeester, 586 A. Goossens, J. Memelink, D. Werck-Reichert, The seco-iridoid pathway from Catharanthus 587 roseus, Nature Communications 5 (2014) 3606-3616.

588

589

590 S. Mortensen, D. Bernal-Franco, L.F. Cole, S. Sathitloetsakun, E.R. Cram, C.W.T. Lee-Parsons, EAS1 transformation: an efficient transient expression method for analyzing gene function in

591 Catharanthus roseus seedlings, Frontiers in Plant Science 11 (2019a) 00755.

592

593 S. Mortensen, J.D. Weaver, S. Sathitloetsakun, L.F. Cole, N.F. Rizvi, E.J. Cram, C.W.T. Lee-

594

595 Parsons, The regulation of $Z C T 1$, a transcriptional repressor of monoterpenoid alkaloid 596 biosynthetic genes in Catharanthus roseus, Plant Direct 3 (2019b) e00193

598

J. Murata, J. Roepke, H. Gordon, V. De Luca, The leaf epidermome of Catharanthus roseus reveals its biochemical specialization, Plant Cell 20 (2008) 524-542.

599

600 Q. Pan, Q. Wang, F. Yuan, S. Xing, J. Zhao, Y.H. Choi, R. Verpoorte, Y. Tian, G. Wang, K.

601 Tang, Overexpression of ORCA3 and G10H in Catharanthus roseus plants regulated alkaloid

602 biosynthesis and metabolism revealed by NMR-metabolomics, PLOS One 7 (2012) e43038.

603

604

G. Pasquali, O.J.M. Goddijn, A. de Waal, R. Verpoorte, R.A. Schilperoort, J.H.C. Hoge, J.

605 Memelink, Coordinated regulation of two indole alkaloid biosynthetic genes from Catharanthus 606 roseus by auxin and elicitors, Plant Molecular Biology 18 (1992) 1121-1131.

607

608 B. Pauw, F.A.O. Hilliou, V.S. Martin, G. Chatel, C.J.F. de Wolf, A. Champion, M. Pré, B. van 609 Duijn, J.W. Kijne, L. van der Fits, J. Memelink, Zinc finger proteins act as transcriptional 610 repressors of alkaloid biosynthesis genes in Catharanthus roseus, Journal of Biological 611 Chemistry 279 (2004) 52940-52948.

612

613 C.A.M. Peebles, E.H. Hughes, J.V. Shanks, K.Y. San, Transcriptional response of the terpenoid 614 indole alkaloid pathway to the overexpression of ORCA3 along with jasmonic acid elicitation of 615 Catharanthus roseus hairy roots over time, Metabolic Engineering 11 (2009) 76-86.

616 
617 M. Pré, Y. Sibéril, J. Memelink, A. Champion, P. Doireau, P. Gantet, Isolation by the yeast one618 hybrid system of cdnas encoding transcription factors that bind to the g-box element of the 619 strictosidine synthase gene promoter from Catharanthus roseus, International Journal of 620 Biochromatography 5 (2000), 229-244.

621

622 N.F. Rizvi, J.D. Weaver, E.J. Cram, C.W.T Lee-Parsons, Silencing the transcriptional repressor, 623 ZCT1, illustrates the tight regulation of terpenoid indole alkaloid biosynthesis in Catharanthus 624 roseus hairy roots, PLOS ONE 11 (2016) e0159712.

625

626 S. Sazegari, A. K. Niazi, F. S. Ahmadi, N. Moshtaghi, G. Younes, CrMYC1 transcription factor 627 overexpression promotes the production of low abundance terpenoid indole alkaloids in 628 Catharanthus roseus, Plant Omics 11 (2018) 30-36.

629

630 F. Schweizer, M. Colinas, J. Pollier, A. Van Moerkercke, R. Vanden Bossche, R. de Clercq, A. Goossens, An engineered combinatorial module of transcription factors boosts production of monoterpenoid indole alkaloids in Catharanthus roseus, Metabolic Engineering 48 (2018) 150633162.

634

635 J.V. Shanks, Phytochemical engineering: combining chemical reaction engineering with plant 636 science, American Institute of Chemical Engineers Journal 51 (2005) 2-7.

637

638 Y. Sibéril, S. Benhamron, J. Memelink, N. Giglioli-Guivarc'h, Catharanthus roseus G-box

639 binding factors 1 and 2 act as repressors of strictosidine synthase gene expression in cell cultures, 640 Plant Molecular Biology 45 (2001) 477-488.

641

642 B. St-Pierre, P. Laflamme, A.M. Alarco, V. De Luca, The terminal O-acetyltransferase involved 643 in vindoline biosynthesis defines a new class of proteins responsible for coenzyme A-dependent 644 acyl transfer, The Plant Journal 14 (1998) 703-713.

645

646 N. Suttipanta, Characterization of $G 10 H$ promoter and characterization of WRKY transcription 647 factors involved in Catharanthus terpenoid indole alkaloid biosynthesis pathway, (2011) D. Phil. 648 Thesis, University of Kentucky.

649

650 N. Suttipanta, S. Pattanaik, M. Kulshrestha, B. Patra, S.K. Singh, L. Yuan, The transcription 651 factor CrWRKY1 positivity regulates indole alkaloid biosynthesis in Catharanthus roseus, Plant Physiology 157 (2011) 2081-2093.

653

654 L. van der Fits, J. Memelink, ORCA3, a jasmonate-responsive transcriptional regulator of plant 655 primary and secondary metabolism, Science 289 (2000) 295-297. 
657 L. van der Fits, H. Zhang, F.L.H. Menke, M. Deneka, J. Memelink, A Catharanthus roseus BPF6581 homologue interacts with an elicitor-responsive region of the secondary metabolite

659 biosynthetic gene Str and is induced by elicitor via a JA-dependent signal transduction pathway, 660 Plant Molecular Biology 44 (2000) 675-685.

661

662

R. van der Heijden, D.I. Jacobs, W. Snoeijer, D. Hallard, R. Verpoorte, The Catharanthus

663 alkaloids: pharmacognosy and biotechnology, Current Medicinal Chemistry 11 (2004) 607-628.

664

665

A. Van Moerkercke, P. Steensma, I. Gariboldi, J. Espoz, P.C. Purnama, F. Schweizer, K.

666 Miettinen, R. Vanden Bossche, J. Memelink, A. Goossens, The basis helix-loop-helix

667 transcription factor BIS2 is essential for monoterpenoid indole alkaloid production in the

668 medicinal plant Catharanthus roseus, The Plant Journal 88 (2016) 3-12.

669

670 A. Van Moerkercke, P. Steensma, F. Schweizer, J. Pollier, I. Gariboldi, R. Payne, R. Vanden 671 Bossche, K. Miettinen, J. Espoz, P.C. Purnama, F. Kellner, T. Seppänen-Laaskso, S.E.

672 O’Connor, H. Rischer, J. Memelink, A. Goossens, The bHLH transcription factor BIS1 controls 673 the iridoid branch of the monoterpenoid indole alkaloid pathway in Catharanthus roseus,

674 Proceedings of the National Academy of Sciences USA 112 (2015) 8130-8135.

675

676 F. Vazquez-Flota, E. De Carolis, A.M. Alarco, V. De Luca, Molecular cloning and

677 characterization of desacetoxyvindoline-4-hydroxylase, a 2-oxoglutarate dependent dioxygenase 678 involved in the biosynthesis of vindoline in Catharanthus roseus (L) G. Don, Plant Molecular 679 Biology 34 (1997) 935-948.

680

681 S. Wei, Methyl jasmonic acid induced expression pattern of terpenoid indole alkaloid pathway

682

683

684 A. Wielopolska, H. Townley, I. Moore, P. Waterhouse, C. Helliwell, A high-throughput 685 inducible RNAi vector for plants, Plant Biotechnology Journal 3 (2005) 583-590.

686

687 H. Zhang, S. Hedhili, G. Montiel, Y. Zhang, G. Chatel, M. Pré, P. Gantet, J. Memelink, The 688 basic helix-loop-helix transcription factor CrMYC2 controls the jasmonate-responsive 689 expression of the ORCA genes that regulate alkaloid biosynthesis in Catharanthus roseus, The 690 Plant Journal 67 (2011) 61-71.

691

692 J. Zuo, Q.W. Niu, N.H. Chua, An estrogen receptor-based transactivator XVE mediates highly 693 inducible gene expression in transgenic plants, The Plant Journal 24 (2000) 265-273. 


\section{Table $\mathbf{1}$ (on next page)}

Correlations between expression of ZCT1, ZCT2 and ZCT3 and expression of TIA biosynthetic and regulatory genes

The Pearson product moment correlation coefficient was determined for the transcript levels of each gene analyzed with the transcript levels of the ZCT1, ZCT2 or ZCT3 genes in the same hairy root line grown for the same amount of time on the same media. $P$ values indicate the significance of the Pearson product moment correlation coefficient $(N=33)$, with values below 0.05 considered to be statistically significant. Bold font indicates a statistically significant positive correlation in gene expression between the two genes being compared. Italicized font indicates a statistically significant negative correlation in gene expression between the two genes being compared. 
1

\begin{tabular}{|c|c|c|c|c|c|c|}
\hline \multirow[b]{2}{*}{$\begin{array}{l}\text { Genes } \\
\text { being } \\
\text { compared }\end{array}$} & \multicolumn{3}{|c|}{ Pearson correlation coefficient } & \multicolumn{3}{|l|}{$P$ value } \\
\hline & ZCT1 & ZCT2 & ZCT3 & $Z C T 1$ & $Z C T 2$ & ZCT3 \\
\hline$C P R$ & 0.62 & 0.49 & 0.36 & 0.00014 & 0.0039 & 0.039 \\
\hline$L A M T$ & 0.75 & $\mathbf{0 . 8 8}$ & 0.85 & $<.00001$ & $<.00001$ & $<.00001$ \\
\hline$T D C$ & 0.73 & 0.66 & 0.52 & $<.00001$ & 0.000033 & 0.0022 \\
\hline STR & 0.57 & 0.56 & 0.54 & 0.00048 & 0.00063 & 0.0011 \\
\hline$T 19 H$ & -0.47 & -0.48 & -0.44 & 0.0064 & 0.0043 & 0.0098 \\
\hline $160 M T$ & 0.61 & 0.59 & 0.42 & 0.00018 & 0.00032 & 0.015 \\
\hline$D 4 H$ & 0.77 & 0.77 & 0.68 & $<.00001$ & $<.00001$ & 0.000014 \\
\hline$D A T$ & 0.85 & 0.93 & 0.86 & $<.00001$ & $<.00001$ & $<.00001$ \\
\hline ORCA2 & -0.27 & -0.29 & -0.44 & 0.12 & 0.11 & 0.011 \\
\hline$O R C A 3$ & -0.20 & -0.47 & -0.25 & 0.28 & 0.0060 & 0.17 \\
\hline$C r B P F 1$ & 0.24 & 0.35 & 0.32 & 0.18 & 0.046 & 0.067 \\
\hline BIS1 & 0.72 & 0.77 & 0.59 & $<.00001$ & $<.00001$ & 0.00031 \\
\hline CrMYC1 & 0.65 & 0.54 & 0.47 & 0.000050 & 0.0011 & 0.0060 \\
\hline CrMYC2 & -0.02 & -0.15 & 0.02 & 0.92 & 0.40 & 0.93 \\
\hline$C r W R K Y 1$ & 0.36 & 0.35 & 0.37 & 0.041 & 0.046 & 0.035 \\
\hline$C r W R K Y 2$ & 0.36 & 0.30 & 0.14 & 0.039 & 0.091 & 0.45 \\
\hline$G B F 1$ & -0.59 & -0.78 & -0.73 & 0.00033 & $<.00001$ & $<.00001$ \\
\hline$G B F 2$ & 0.62 & 0.56 & 0.55 & 0.00012 & 0.00080 & 0.00090 \\
\hline$Z C T 1$ & $\mathrm{~N} / \mathrm{A}$ & 0.91 & 0.92 & $\mathrm{~N} / \mathrm{A}$ & $<.00001$ & $<.00001$ \\
\hline ZCT2 & 0.91 & $\mathrm{~N} / \mathrm{A}$ & 0.92 & $<.00001$ & N/A & $<.00001$ \\
\hline ZCT3 & 0.92 & 0.92 & N/A & $<.00001$ & $<.00001$ & $\mathrm{~N} / \mathrm{A}$ \\
\hline
\end{tabular}




\section{Figure 1}

TIA biosynthetic pathway in C. roseus.

The metabolites resulting from different biochemical reactions are indicated below the arrows. Solid arrows signify single enzymatic reactions whereas dotted arrows indicate multiple enzymatic reactions. The genes included for analysis are indicated next to the appropriate arrows. 


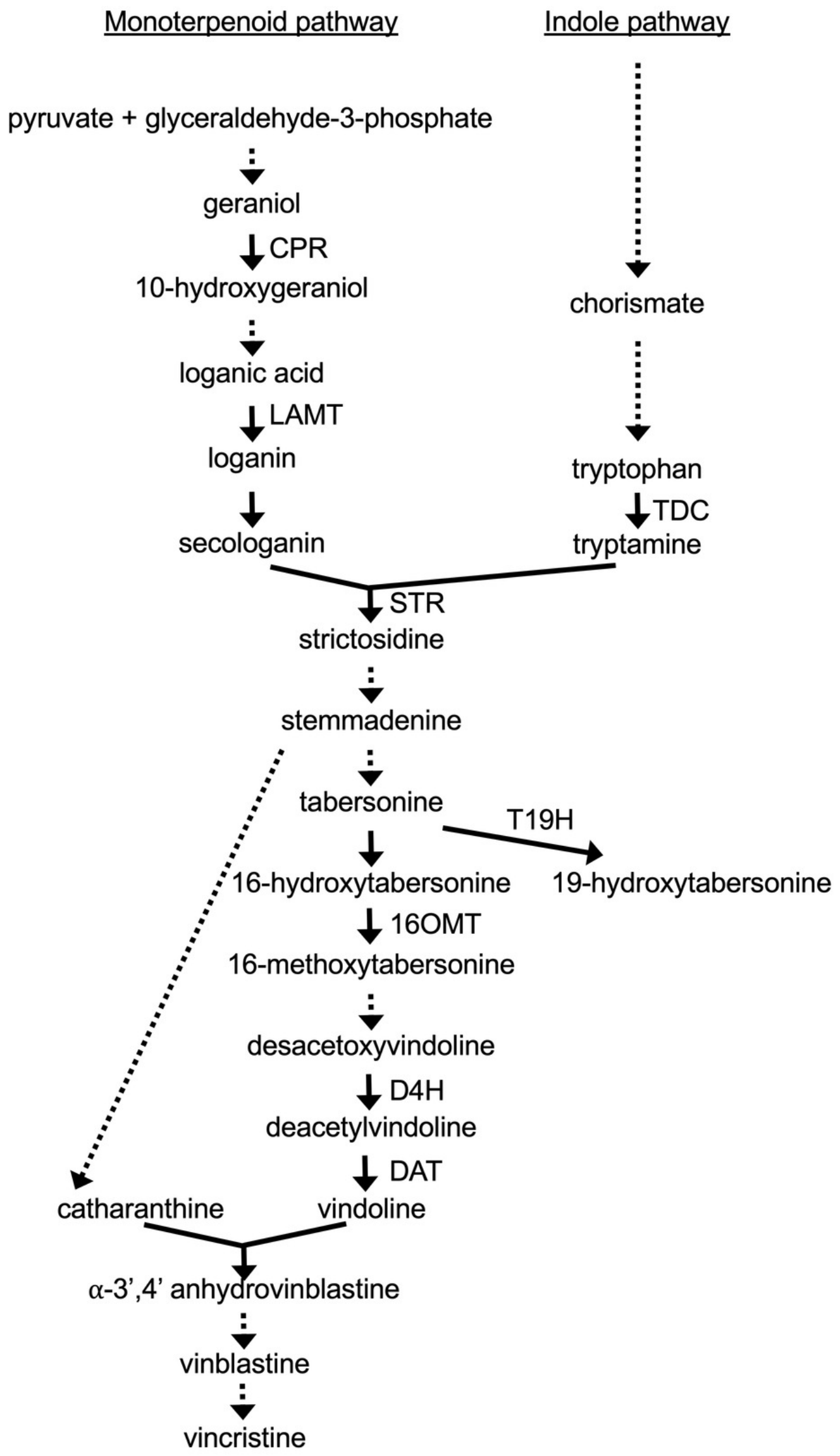




\section{Figure 2}

C. roseus transgenic hairy root lines carrying the XPZ RNAi construct have decreased ZCT transcript levels.

(A) ZCT1, (B) ZCT2 and (C) ZCT3 transcript levels were assayed in the XPZ28 (28) and XPZ38 (38) transgenic hairy root lines, which carry the XPZ RNAi construct designed to reduce expression of all three $Z C T$ genes. $Z C T$ transcript levels were also analyzed in a control line (C), transformed with the XVE-pOpOff2 empty vector. Cultures from all three hairy root lines were treated with $0 \mu \mathrm{M}(0)$ or $20 \mu \mathrm{M}(20)$ beta-estradiol and then harvested after the indicated number of hours. The results of Student's T-tests, comparing different lines or treatment conditions at different time points, are shown below each graph. T-test results below 0.05 are highlighted by bold font. Results are the average $\Delta \Delta C T$ value of three biological replicates, with two technical replicates per biological replicate. Error bars indicate standard deviations. 


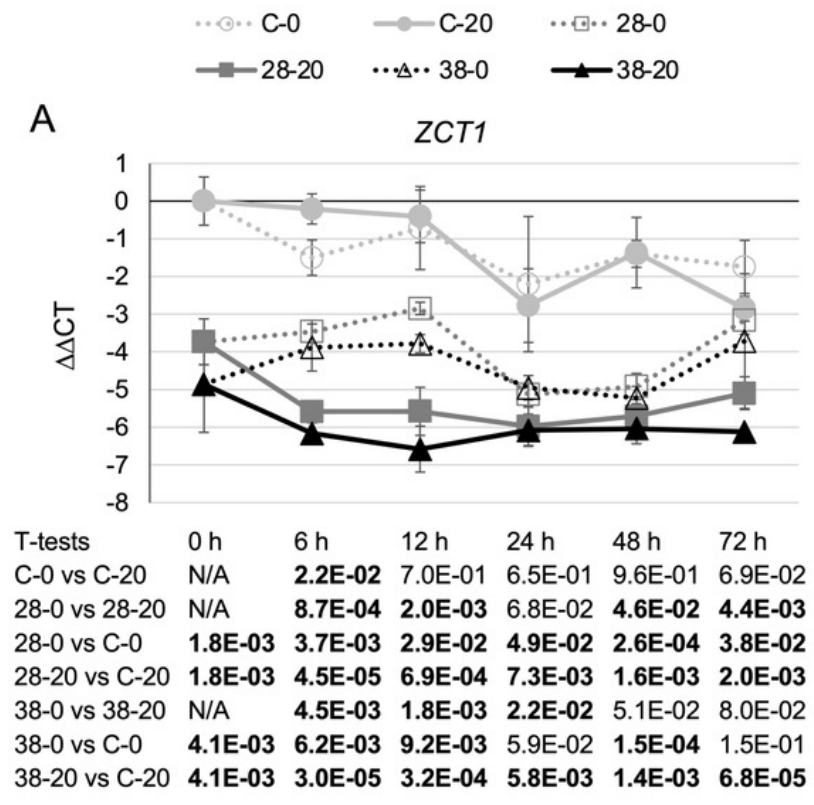

B

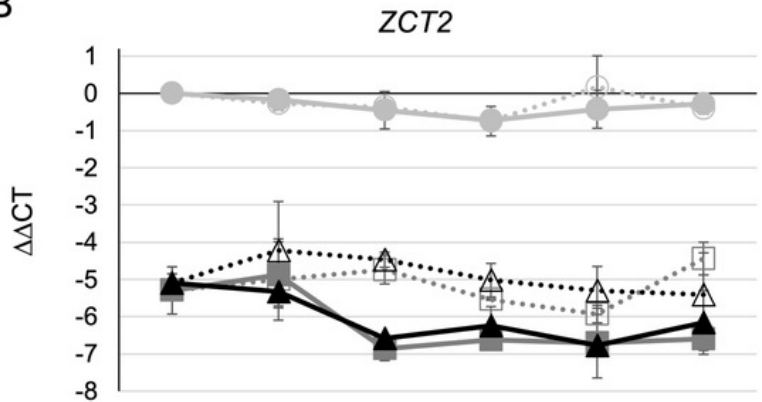

$\begin{array}{lllllll}\text { T-tests } & 0 \mathrm{~h} & 6 \mathrm{~h} & 12 \mathrm{~h} & 24 \mathrm{~h} & 48 \mathrm{~h} & 72 \mathrm{~h}\end{array}$

C-0 vs C-20 N/A 5.1E-01 7.9E-01 9.4E-01 3.5E-01 4.8E-01

28-0 vs 28-20 N/A $8.8 \mathrm{E}-01 \quad 1.8 \mathrm{E}-03 \quad 2.2 \mathrm{E}-03 \quad 2.3 \mathrm{E}-01 \quad 2.2 \mathrm{E}-03$

28-0 vs C-0 1.6E-04 1.8E-03 4.8E-05 3.1E-05 2.7E-04 1.1E-04

28-20 vs C-20 1.6E-04 8.1E-04 4.9E-05 9.9E-06 5.2E-04 8.8E-06

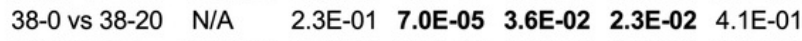

38-0 vs C-0 9.8E-06 1.1E-02 7.3E-06 2.5E-04 8.7E-04 1.6E-03

$38-20$ vs C-20 9.8E-06 2.4E-05 3.1E-05 7.5E-05 4.9E-05 3.3E-04

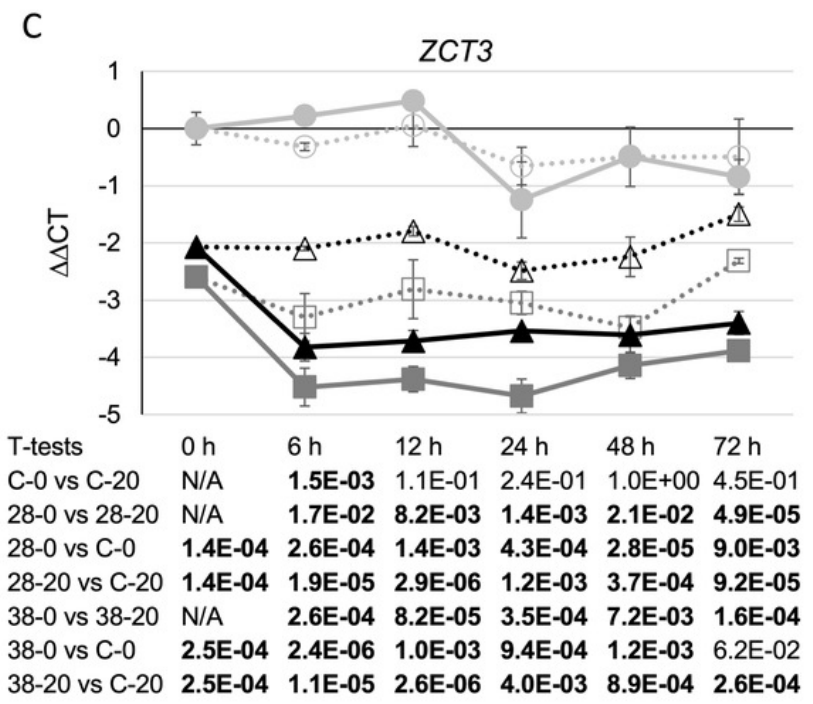

Peer) reviewing PDF | (2021:01:57402:1:1:NEW 6 May 2021) 


\section{Figure 3}

Time course of CPR and LAMT expression.

(A) CPR and (B) LAMT transcript levels were assayed in the XPZ28 (28), XPZ38 (38) and control lines (C) grown on $0 \mu \mathrm{m}(0)$ or $20 \mu \mathrm{M}(20)$ beta-estradiol and harvested at the indicated time points. The results of Student's T-tests, comparing different lines or treatment conditions at different time points, are shown below each graph. T-test results below 0.05 are highlighted by bold font. Results are the average $\Delta \Delta C T$ value of three biological replicates, with two technical replicates per biological replicate. Error bars indicate standard deviations. 

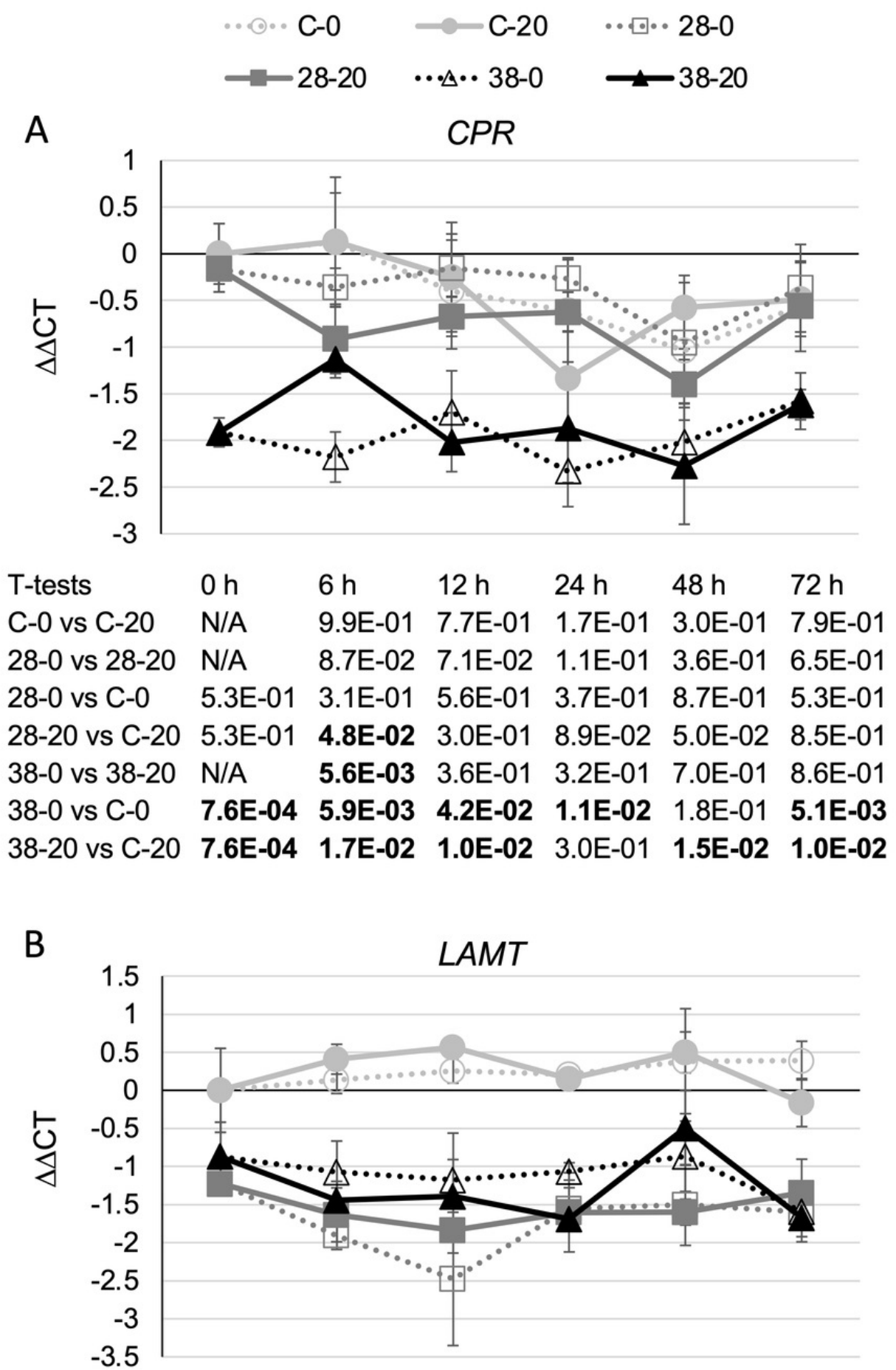

\begin{tabular}{|c|c|c|c|c|c|c|}
\hline ts & $\mathrm{Oh}$ & $6 \mathrm{~h}$ & $12 \mathrm{~h}$ & $24 \mathrm{~h}$ & $48 \mathrm{~h}$ & $72 \mathrm{~h}$ \\
\hline s C-20 & N/A & $5 E-01$ & 3.3E-02 & 3.6E-01 & 0E-01 & \\
\hline & N/A & & 3.0E-01 & & & \\
\hline & & & & & & \\
\hline C-2 & 2.1E-( & 4E-04 & 1.6E-04 & $.6 E-03$ & -04 & $=-02$ \\
\hline $8-20$ & $\mathrm{~N} / \mathrm{A}$ & $5 \mathrm{E}-01$ & $6.6 \mathrm{E}-01$ & .4E-03 & 3.9E-01 & $8 \mathrm{E}-01$ \\
\hline $3-0$ vs C- 0 & & $.3 E-03$ & & & & $1.2 \mathrm{E}-03$ \\
\hline $3-20$ vs C-20 & 1.0E-01 & $.2 E-04$ & 2.2E-03 & $3.8 \mathrm{E}-05$ & 3.7E-02 & 4.2E-0 \\
\hline
\end{tabular}




\section{Figure 4}

Time course of TDC expression.

TDC transcript levels were assayed in the XPZ28 (28), XPZ38 (38) and control lines (C) grown on $0 \mu \mathrm{m}(0)$ or $20 \mu \mathrm{M}(20)$ beta-estradiol and harvested at the indicated time points. The results of Student's T-tests, comparing different lines or treatment conditions at different time points, are shown below each graph. T-test results below 0.05 are highlighted by bold font. Results are the average $\Delta \triangle C T$ value of three biological replicates, with two technical replicates per biological replicate. Error bars indicate standard deviations. 


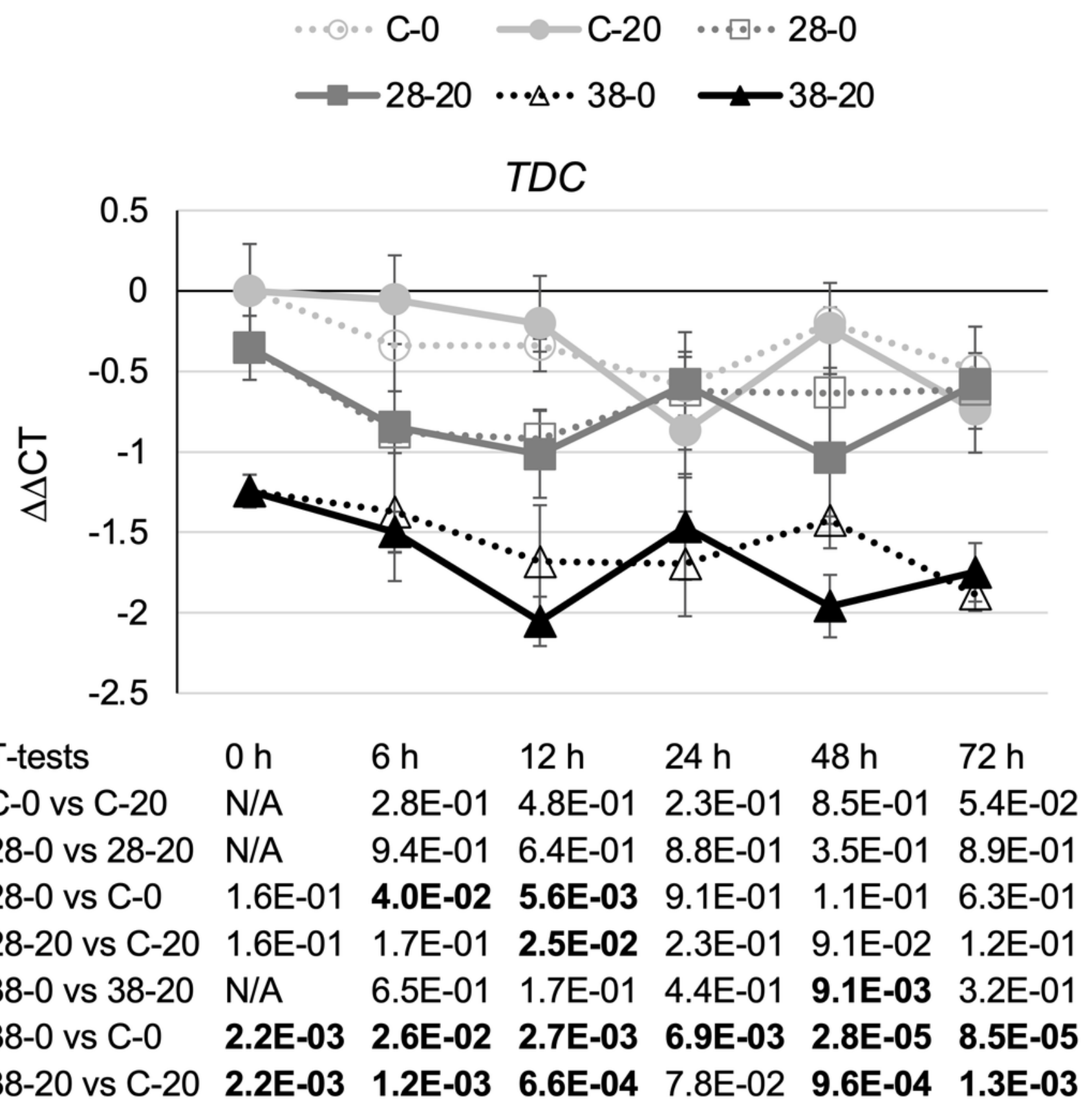




\section{Figure 5}

Time course of expression of TIA biosynthetic genes.

(A) STR, (B) T19H, (C) 16OMT, (D) D4H and (E) DAT transcript levels were assayed in the XPZ28 (28), XPZ38 (38) and control lines (C) grown on $0 \mu \mathrm{m}(0)$ or $20 \mu \mathrm{M}$ (20) beta-estradiol and harvested at the indicated time points. The results of Student's T-tests, comparing different lines or treatment conditions at different time points, are shown below each graph. T-test results below 0.05 are highlighted by bold font. Results are the average $\Delta \Delta C T$ value of three biological replicates, with two technical replicates per biological replicate. Error bars indicate standard deviations. 


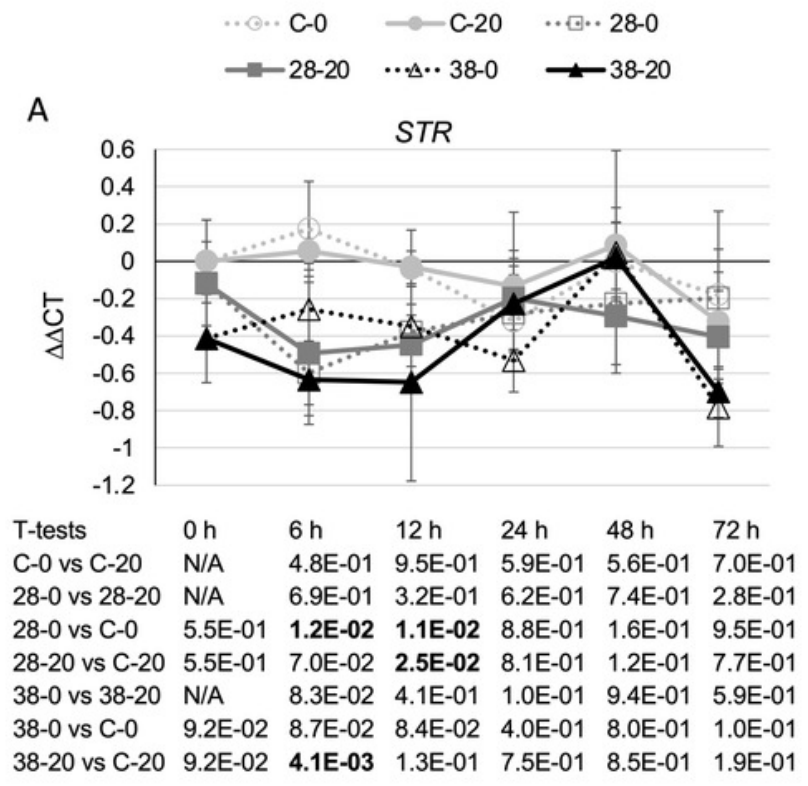

B

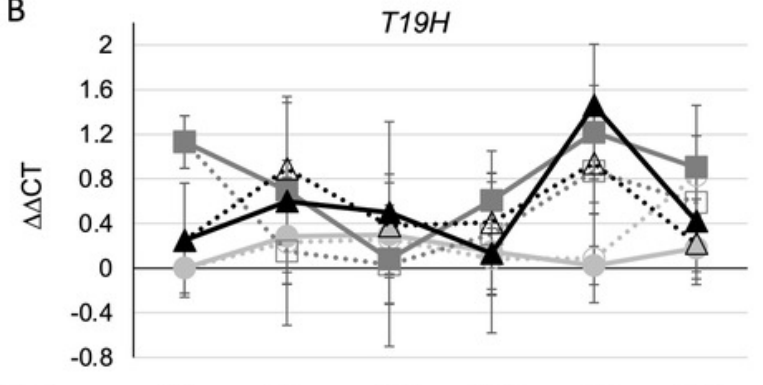

T-tests

$0 \mathrm{~h} \quad 6 \mathrm{~h} \quad 12 \mathrm{~h} \quad 24 \mathrm{~h} \quad 48 \mathrm{~h} \quad 72 \mathrm{~h}$

$\mathrm{C}-0$ vs $\mathrm{C}-20 \quad$ N/A $\quad 8.6 \mathrm{E}-01 \quad 8.4 \mathrm{E}-01 \quad 8.3 \mathrm{E}-01 \quad 8.2 \mathrm{E}-01 \quad 1.8 \mathrm{E}-01$ 28-0 vs 28-20 N/A 4.3E-01 9.2E-01 4.2E-01 3.5E-01 2.3E-01 28-0 vs C-0 3.8E-03 8.5E-01 6.5E-01 5.7E-01 6.9E-02 5.7E-01 28-20 vs C-20 3.8E-03 4.9E-01 4.3E-01 1.3E-01 $\quad$ 1.0E-02 $3.4 \mathrm{E}-02$ $38-0$ vs 38-20 N/A $5.1 \mathrm{E}-01 \quad 8.4 \mathrm{E}-01 \quad 6.5 \mathrm{E}-01 \quad 2.4 \mathrm{E}-01 \quad 6.1 \mathrm{E}-01$ 38-0 vs C-0 4.8E-01 1.6E-01 7.2E-01 4.7E-01 5.0E-02 2.0E-01 38-20 vs C-20 4.8E-01 3.9E-01 7.0E-01 9.8E-01 1.2E-02 5.4E-01

C

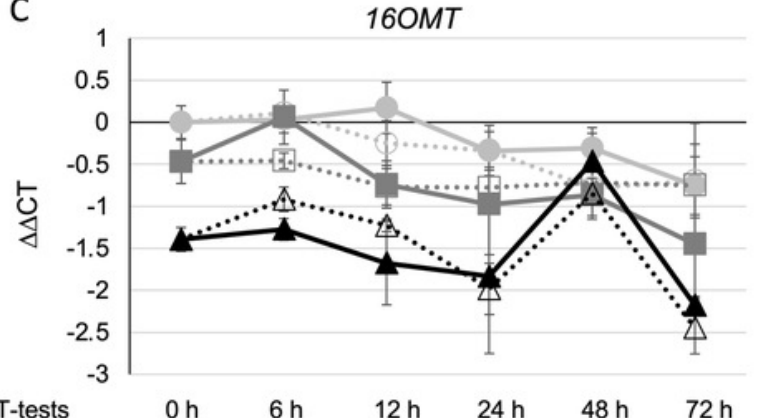

$\begin{array}{llllll}\mathrm{C}-0 \text { vs C-20 N/A } & 4.8 \mathrm{E}-01 & 1.5 \mathrm{E}-01 & 9.8 \mathrm{E}-01 & 3.8 \mathrm{E}-02 & 8.7 \mathrm{E}-01\end{array}$ 28-0 vs 28-20 N/A 5.5E-02 9.0E-01 6.2E-01 5.6E-01 2.8E-01 $28-0$ vs C-0 6.7E-02 $1.0 \mathrm{E}-03 \quad 5.8 \mathrm{E}-02 \quad 1.2 \mathrm{E}-01 \quad 8.0 \mathrm{E}-01 \quad 9.2 \mathrm{E}-01$ $28-20$ vs C-20 6.7E-02 9.0E-01 1.9E-02 1.6E-01 1.7E-02 1.6E-01 $38-0$ vs 38-20 N/A $\quad 3.4 \mathrm{E}-02 \quad 2.0 \mathrm{E}-01 \quad 8.0 \mathrm{E}-01 \quad 2.2 \mathrm{E}-01 \quad 2.2 \mathrm{E}-01$ 38-0 vs C-0 5.6E-04 3.7E-04 3.5E-03 2.6E-03 7.4E-01 4.8E-03 38-20 vs C-20 5.6E-04 3.9E-04 5.4E-03 5.3E-02 5.5E-01 2.2E-03
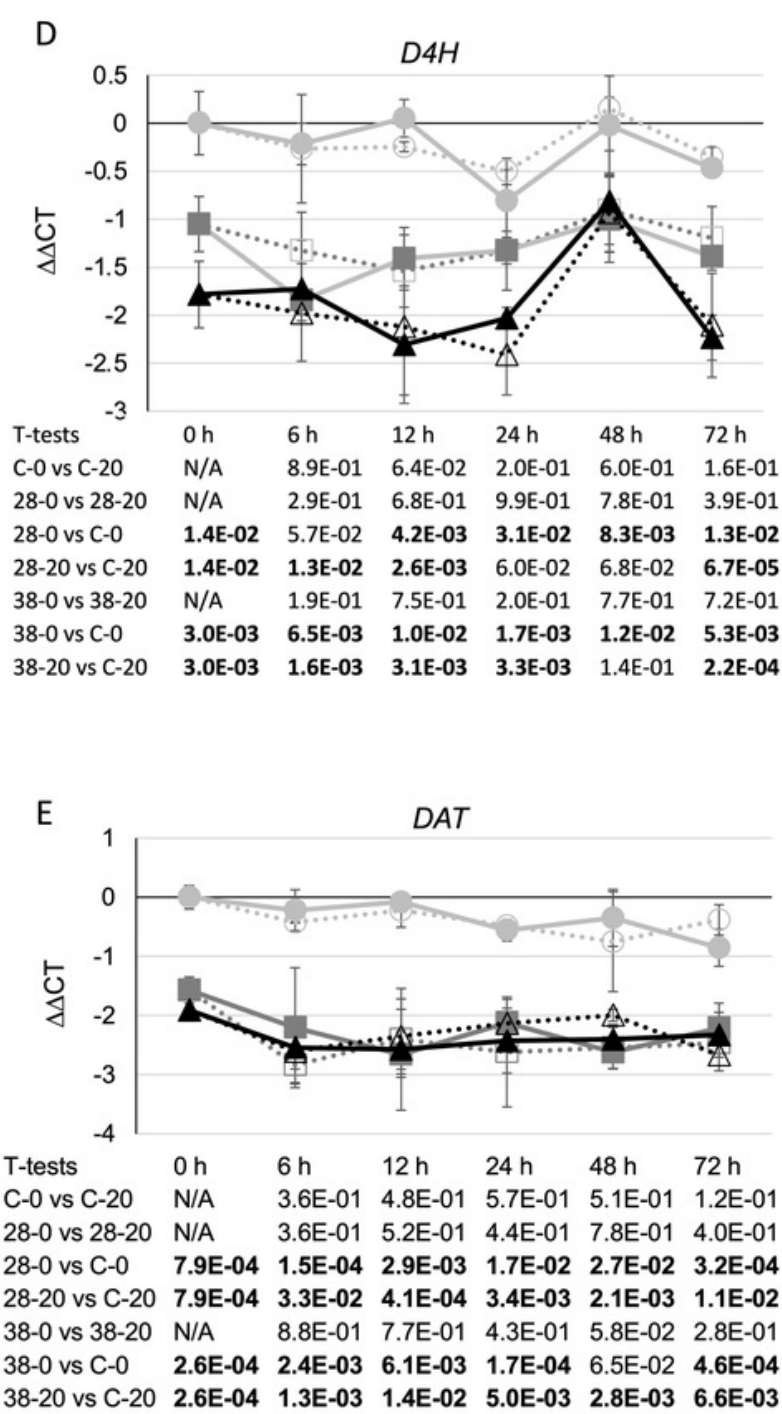


\section{Figure 6}

Time course of expression of TIA positive transcriptional regulators.

(A) ORCA2, (B) ORCA3, (C) BIS1, (D) CrBPF1, (E) CrMYC1, (F) CrMYC2, (G) CrWRKY1 and (H) CrWRKY2 transcript levels were assayed in the XPZ28 (28), XPZ38 (38) and control lines (C) grown on $0 \mu \mathrm{m}(0)$ or $20 \mu \mathrm{M}(20)$ beta-estradiol and harvested at the indicated time points. The results of Student's T-tests, comparing different lines or treatment conditions at different time points, are shown below each graph. T-test results below 0.05 are highlighted by bold font. Results are the average $\Delta \triangle C T$ value of three biological replicates, with two technical replicates per biological replicate. Error bars indicate standard deviations. 


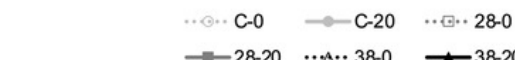

A

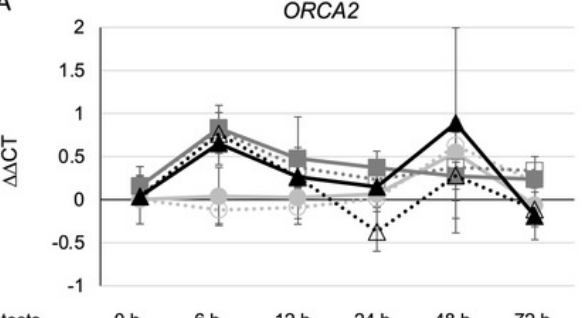

$\begin{array}{lllllll}\text { T-tests } & 0 \mathrm{~h} & 6 \mathrm{~h} & 12 \mathrm{~h} & 24 \mathrm{~h} & 48 \mathrm{~h} & 72 \mathrm{~h}\end{array}$ $\begin{array}{lllllll}\mathrm{C}-0 \text { vs C-20 } & \text { N/A } & 5.1 \mathrm{E}-01 & 5.7 \mathrm{E}-01 & 4.8 \mathrm{E}-01 & 7.4 \mathrm{E}-01 & 1.4 \mathrm{E}-01 \\ 28-0 \text { vs } 28-20 & \text { N/A } & 9.1 \mathrm{E}-01 & 7.7 \mathrm{E}-01 & 4.5 \mathrm{E}-01 & 8.2 \mathrm{E}-01 & 3.8 \mathrm{E}-01\end{array}$ 28-0 vs C-0 $\quad 4.9 \mathrm{E}-01 \quad 3.5 \mathrm{E}-03 \quad 5.5 \mathrm{E}-02 \quad 1.7 \mathrm{E}-01 \quad 2.2 \mathrm{E}-01 \quad 3.1 \mathrm{E}-01$ $28-20$ vs C-20 4.9E-01 3.3E-02 2.2E-01 5.7E-02 $\quad 5.7 \mathrm{E}-01 \quad 1.1 \mathrm{E}-01$ $38-0$ vs $38-20 \quad N / A \quad 5.9 \mathrm{E}-01 \quad 9.8 \mathrm{E}-01 \quad 7.0 \mathrm{E}-02 \quad 4.1 \mathrm{E}-01 \quad 7.0 \mathrm{E}-01$

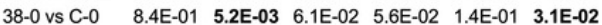

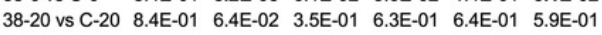

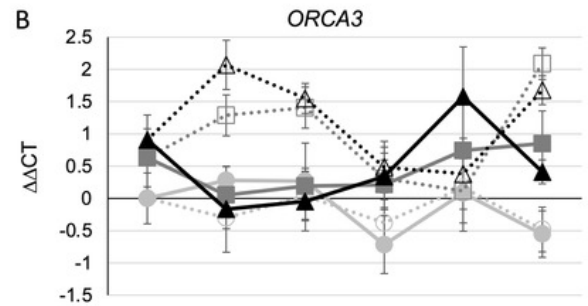

$\begin{array}{lllllll}\text { T-tests } & 0 \mathrm{~h} & 6 \mathrm{~h} & 12 \mathrm{~h} & 24 \mathrm{~h} & 48 \mathrm{~h} & 72 \mathrm{~h}\end{array}$ $\begin{array}{llllll}\mathrm{C}-0 \text { vs C-20 N/A } & 2.1 \mathrm{E}-02 & 6.0 \mathrm{E}-01 & 3.1 \mathrm{E}-01 & 8.2 \mathrm{E}-01 & 8.2 \mathrm{E}-01\end{array}$

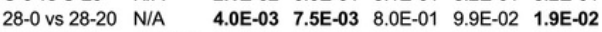

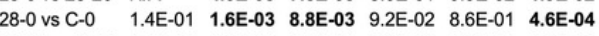
28-20 vs C-20 1.4E-01 2.2E-01 8.5E-01 5.2E-02 $1.3 \mathrm{E}-01 \quad 1.7 \mathrm{E}-02$ $38-0$ vs $38-20 \quad \mathrm{~N} / \mathrm{A} \quad 7.3 \mathrm{E}-03 \quad 5.7 \mathrm{E}-03 \quad 6.7 \mathrm{E}-01 \quad 9.5 \mathrm{E}-02 \quad 1.7 \mathrm{E}-03$

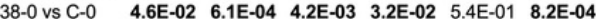
$38-20$ vs C-20 4.6E-02 3.3E-01 5.1E-01 3.3E-02 5.7E-02 $1.5 \mathrm{E}-02$
C

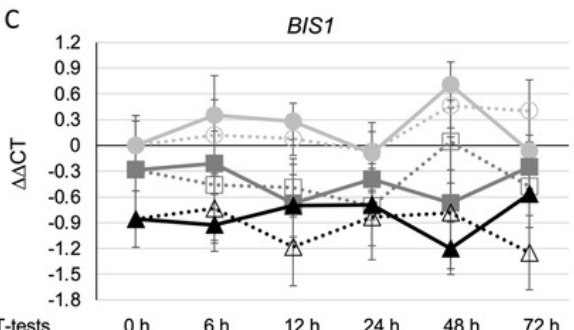

$\begin{array}{lllllll}\text { T-tests } & 0 \mathrm{~h} & 6 \mathrm{~h} & 12 \mathrm{~h} & 24 \mathrm{~h} & 48 \mathrm{~h} & 72 \mathrm{~h}\end{array}$ $\begin{array}{lllllll}\mathrm{C}-0 \text { vs C- } 20 & \text { N/A } & 2.6 \mathrm{E}-01 & 3.0 \mathrm{E}-01 & 9.3 \mathrm{E}-01 & 3.2 \mathrm{E}-01 & 1.2 \mathrm{E}-01 \\ 28-0 \text { vs } 28-20 & \mathrm{~N} / \mathrm{A} & 6.9 \mathrm{E}-01 & 6.1 \mathrm{E}-01 & 5.6 \mathrm{E}-02 & 2.4 \mathrm{E}-01 & 2.7 \mathrm{E}-01\end{array}$

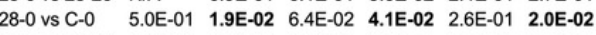
28-20 vs C-20 5.0E-01 4.0E-01 3.0E-02 1.4E-01 4.3E-02 3.6E-01

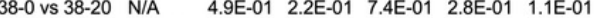

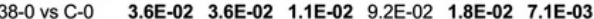

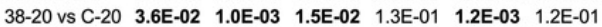

D

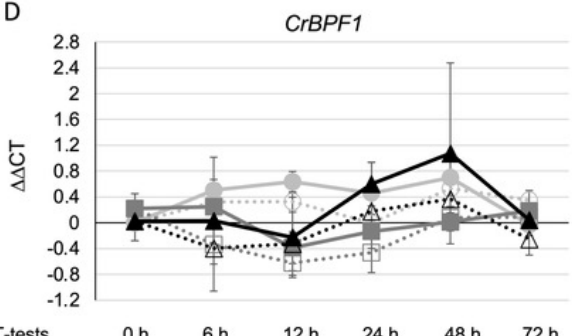

$\begin{array}{lllllll} & 0 \mathrm{~h} & 6 \mathrm{~h} & 12 \mathrm{~h} & 24 \mathrm{~h} & 48 \mathrm{~h} & 72 \mathrm{~h}\end{array}$ $\begin{array}{lllllll}\mathrm{C}-0 \text { vs C-20 N/A } & 6.4 \mathrm{E}-01 & 5.8 \mathrm{E}-02 & 1.7 \mathrm{E}-01 & 6.4 \mathrm{E}-01 & 4.5 \mathrm{E}-02\end{array}$ $\begin{array}{lllllll}28-0 \text { vs } 28-20 & \text { N/A } & 8.6 \mathrm{E}-02 & 2.1 \mathrm{E}-01 & 2.4 \mathrm{E}-01 & 6.5 \mathrm{E}-01 & 4.5 \mathrm{E}-01 \\ 28-0 \text { vs C-0 } & 3.5 \mathrm{E}-01 & 6.9 \mathrm{E}-02 & 7.1 \mathrm{E}-04 & 6.7 \mathrm{E}-02 & 2.2 \mathrm{E}-01 & 1.0 \mathrm{E}-01\end{array}$

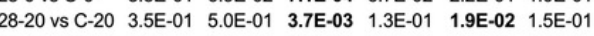
$38-0$ vs $38-20 \quad N / A \quad 3.8 \mathrm{E}-01 \quad 8.4 \mathrm{E}-01 \quad 1.7 \mathrm{E}-01 \quad 4.5 \mathrm{E}-01 \quad 1.5 \mathrm{E}-01$

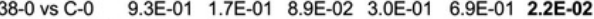
$38-20$ vs C-20 9.3E-01 2.6E-01 8.0E-02 $\quad 7.0 \mathrm{E}-01 \quad 6.7 \mathrm{E}-01 \quad 6.3 \mathrm{E}-01$

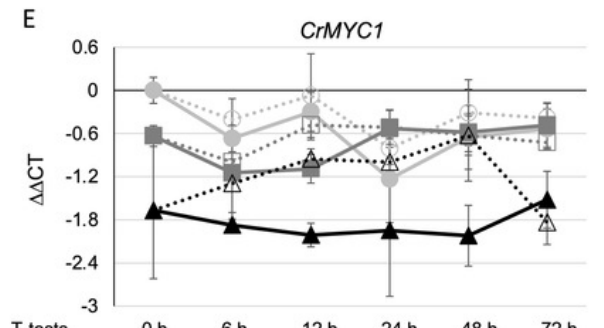

$\begin{array}{lllllll}\text { T-tests } & 0 \mathrm{~h} & 6 \mathrm{~h} & 12 \mathrm{~h} & 24 \mathrm{~h} & 48 \mathrm{~h} & 72 \mathrm{~h} \\ \mathrm{C}-0 \text { vs C-20 } & \text { N/A } & 2.6 \mathrm{E}-01 & 5.7 \mathrm{E}-01 & 3.0 \mathrm{E}-01 & 4.1 \mathrm{E}-01 & 5.0 \mathrm{E}-01\end{array}$ $\begin{array}{llllllll}28-0 & \text { vs } 28-20 & \text { N/A } & 6.6 \mathrm{E}-01 & 2.1 \mathrm{E}-02 & 9.4 \mathrm{E}-01 & 9.3 \mathrm{E}-01 & 2.6 \mathrm{E}-01\end{array}$ 28-0 vs C-0 9.0E-03 2.6E-02 3.1E-01 1.4E-01 5.3E-01 6.5E-02 28-20 vs C-20 9.0E-03 2.3E-01 1.5E-02 1.4E-01 8.3E-01 8.4E-01

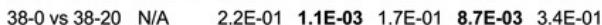
$\begin{array}{lllllll}38-0 \text { vs C-0 } & 4.0 \mathrm{E}-02 & 1.1 \mathrm{E}-01 & 6.3 \mathrm{E}-02 & 4.1 \mathrm{E}-01 & 3.6 \mathrm{E}-01 & 2.6 \mathrm{E}-03\end{array}$

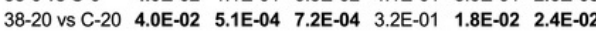

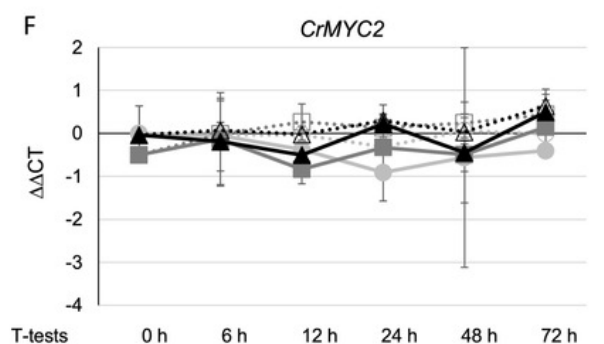

$\begin{array}{lllllll}\text { T-tests } & 0 \mathrm{~h} & 6 \mathrm{~h} & 12 \mathrm{~h} & 24 \mathrm{~h} & 48 \mathrm{~h} & 72 \mathrm{~h}\end{array}$ $\begin{array}{lllllll}\mathrm{C}-0 \text { vs C-20 N/A } & 9.7 \mathrm{E}-01 & 3.6 \mathrm{E}-01 & 2.1 \mathrm{E}-01 & 7.0 \mathrm{E}-01 & 2.1 \mathrm{E}-01\end{array}$

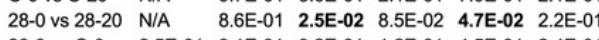
28-0 vs C-0 2.5E-01 9.1E-01 3.2E-01 1.2E-01 4.5E-01 2.4E-01 28-20 vs C-20 2.5E-01 9.2E-01 2.8E-01 2.1E-01 9.6E-01 2.1E-03 38-0 vs 38-20 N/A 6.9E-01 4.2E-02 7.6E-01 5.2E-01 6.9E-01 $\begin{array}{lllllll}38-0 & \text { vs C-0 } & 9.3 \mathrm{E}-01 \quad 4.8 \mathrm{E}-01 & 9.1 \mathrm{E}-01 & 6.4 \mathrm{E}-02 & 9.1 \mathrm{E}-01 & 1.3 \mathrm{E}-01\end{array}$

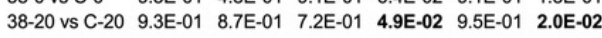

G

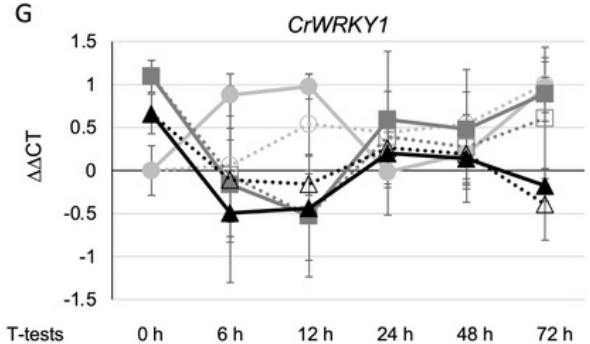

$\begin{array}{lllllll}\text { T-tests } & 0 \mathrm{~h} & 6 \mathrm{~h} & 12 \mathrm{~h} & 24 \mathrm{~h} & 48 \mathrm{~h} & 72 \mathrm{~h} \\ \mathrm{C}-0 \text { vs C-20 } & \text { N/A } & 1.8 \mathrm{E}-01 & 2.7 \mathrm{E}-01 & 3.4 \mathrm{E}-02 & 1.3 \mathrm{E}-01 & 9.1 \mathrm{E}-01\end{array}$ $28-0$ vs $28-20 \quad$ N/A $\quad 8.9 \mathrm{E}-01 \quad 1.0 \mathrm{E}+00 \quad 6.9 \mathrm{E}-01 \quad 7.2 \mathrm{E}-01 \quad 5.4 \mathrm{E}-01$ 28-0 vs C-0 5.1E-03 8.5E-01 7.8E-02 7.5E-01 5.2E-01 4.5E-01 28-20 vs C-20 5.1E-03 2.0E-01 2.3E-02 2.6E-01 5.5E-01 7.3E-01

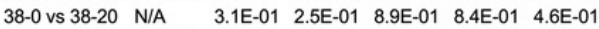

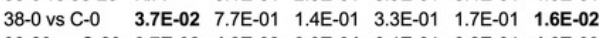

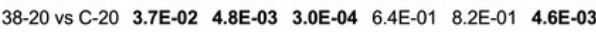

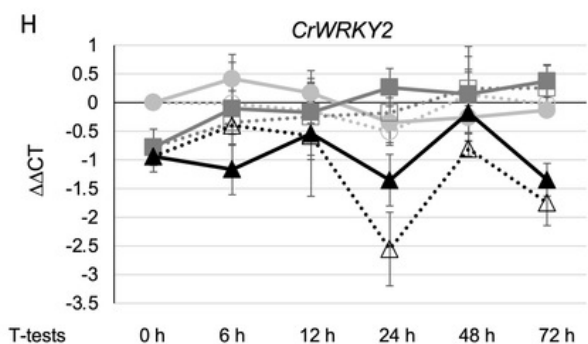

C- 0 vs C-20 N/A $\quad 4.2 \mathrm{E}-01 \quad 3.5 \mathrm{E}-01 \quad 5.4 \mathrm{E}-01 \quad 3.9 \mathrm{E}-01 \quad 2.2 \mathrm{E}-01$ 28-0 vs $28-20$ N/A $6.8 \mathrm{E}-01 \quad 8.5 \mathrm{E}-01 \quad 1.4 \mathrm{E}-01 \quad 8.7 \mathrm{E}-01 \quad 7.0 \mathrm{E}-01$ $28-0$ vs C-0 1.4E-02 6.5E-01 8.4E-01 1.8E-01 8.3E-01 3.1E-01 28-20 vs C-20 1.4E-02 1.6E-01 9.3E-02 9.3E-02 $\quad 4.6 \mathrm{E}-01 \quad 3.4 \mathrm{E}-02$

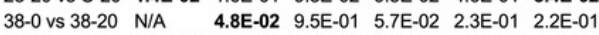

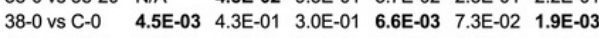
$38-20$ vs C-20 4.5E-03 1.1E-02 $3.3 \mathrm{E}-01 \quad 3.8 \mathrm{E}-02 \quad 8.6 \mathrm{E}-01 \quad 2.0 \mathrm{E}-03$ 


\section{Figure 7}

Time course of expression of TIA negative transcriptional regulators.

(A) GBF1 and (B) GBF2 transcript levels were assayed in the XPZ28 (28), XPZ38 (38) and control lines (C) grown on $0 \mu \mathrm{m}(0)$ or $20 \mu \mathrm{M}(20)$ beta-estradiol and harvested at the indicated time points. The results of Student's T-tests, comparing different lines or treatment conditions at different time points, are shown below each graph. T-test results below 0.05 are highlighted by bold font. Results are the average $\Delta \Delta C T$ value of three biological replicates, with two technical replicates per biological replicate. Error bars indicate standard deviations. 


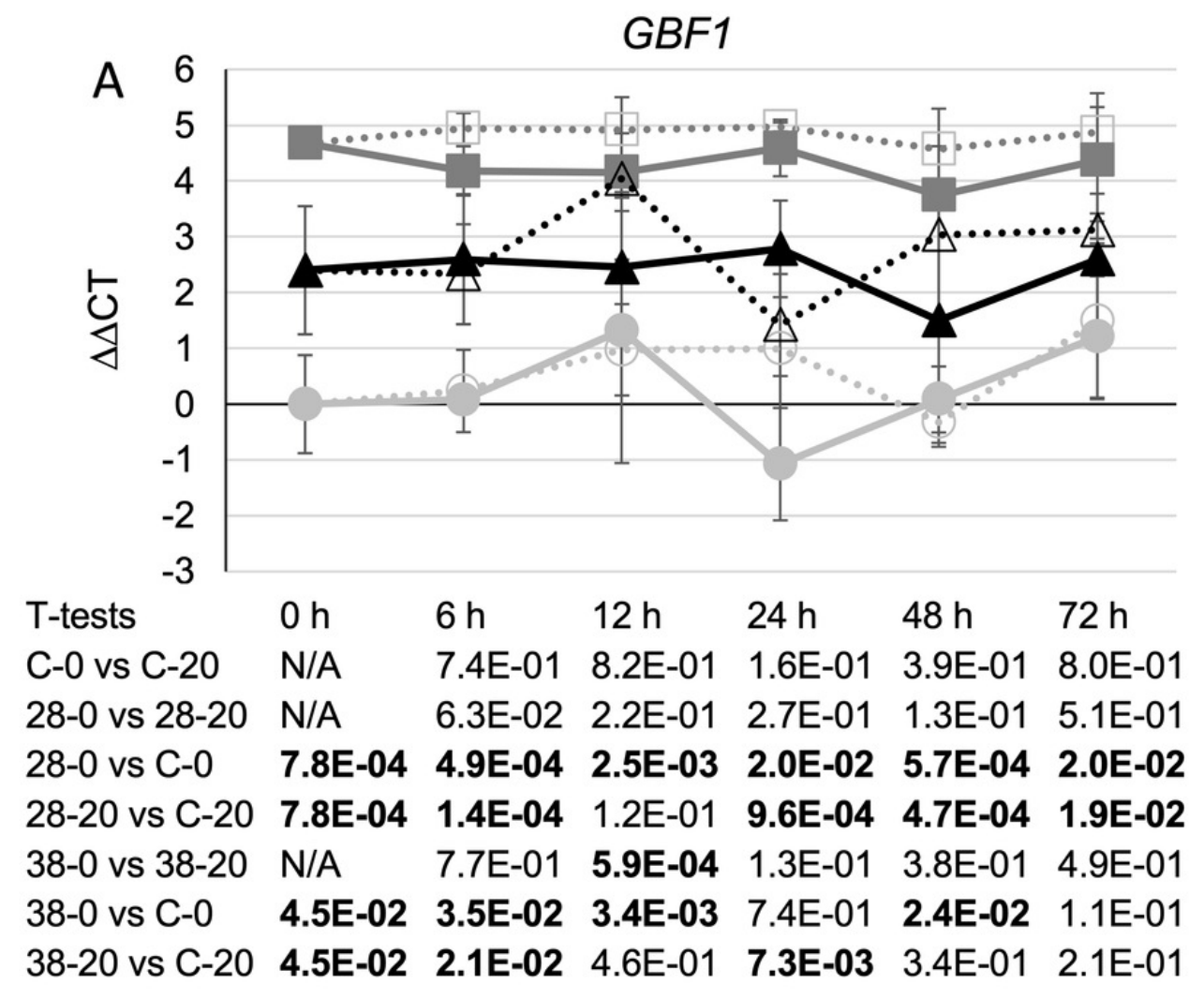

B

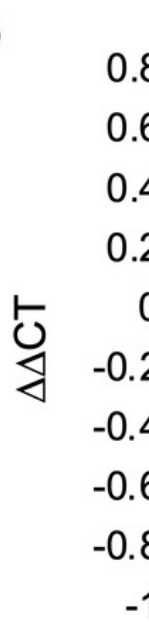

T-tests

$\mathrm{C}-0$ vs $\mathrm{C}-20$

28-0 vs $28-20$

$28-0$ vs C- 0

$28-20$ vs C-20

$38-0$ vs $38-20$

$38-0$ vs C- 0

$38-20$ vs C-20

$\mathrm{Oh}$

$6 \mathrm{~h}$

$12 \mathrm{~h}$

$24 \mathrm{~h}$

$48 \mathrm{~h}$

$72 \mathrm{~h}$

\section{N/A}

$6.3 \mathrm{E}-02$

2.2E-01

2.7E-01

$1.3 \mathrm{E}-01 \quad 5.1 \mathrm{E}-01$

7.8E-04 1.4E-04

2.5E-03

9.6E-04 4.7E-04 1.9E-02

$\mathrm{N} / \mathrm{A}$

7.7E-01

4.5E-02 2.1E-02 4.6E-01

7.3E-03

$3.4 \mathrm{E}-01$

\subsection{E-01}

GBF2

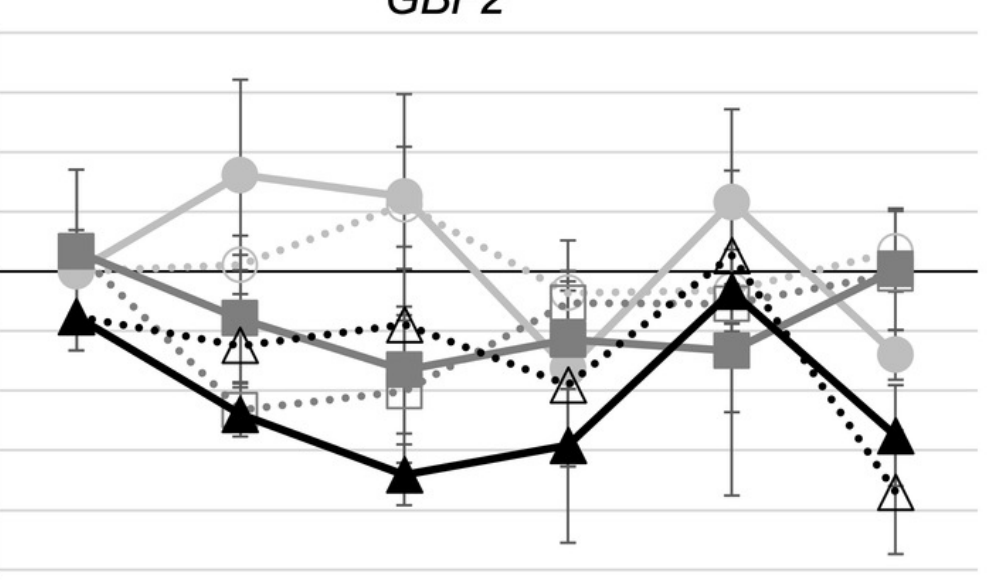

$\begin{array}{llllll}0 \mathrm{~h} & 6 \mathrm{~h} & 12 \mathrm{~h} & 24 \mathrm{~h} & 48 \mathrm{~h} & 72 \mathrm{~h}\end{array}$

N/A $\quad 1.9 \mathrm{E}-01 \quad 9.2 \mathrm{E}-01 \quad 3.0 \mathrm{E}-01 \quad 2.1 \mathrm{E}-01 \quad 2.0 \mathrm{E}-02$

N/A $\quad 8.3 \mathrm{E}-02 \quad 7.2 \mathrm{E}-01 \quad 3.0 \mathrm{E}-01 \quad 6.1 \mathrm{E}-01 \quad 9.0 \mathrm{E}-01$

7.3E-01 3.1E-03 6.9E-02 7.8E-01 6.4E-01 3.9E-01

7.3E-01

$9.5 \mathrm{E}-02$

2.1E-02 6.7E-01

2.1E-01 8.8E-02

N/A

4.8E-02 1.5E-02 4.2E-01

6.7E-01 2.9E-01

2.1E-01

$5.2 \mathrm{E}-02$

$1.7 \mathrm{E}-01 \quad 1.2 \mathrm{E}-01$

$4.8 \mathrm{E}-01$

4.8E-03

2.1E-01

1.2E-02 1.2E-03 3.9E-01

3.7E-01

$6.8 \mathrm{E}-02$ 